\title{
Existence of a strong solution to moist atmospheric equations with the effects of topography
}

\author{
Ruxu Lian ${ }^{1,2^{*}}$ and Jieqiong $\mathrm{Ma}^{3^{*}}$ (B)
}

\author{
"Correspondence: \\ lianruxu@mail.iap.ac.cn; \\ jiegiong.ma@outlook.com \\ ${ }^{1}$ School of Mathematics and \\ Statistics, North China University of \\ Water Resources and Electric Power, \\ Zhengzhou, P.R. China \\ ${ }^{3}$ School of Mathematical Sciences, \\ Capital Normal University, Beijing, \\ P.R. China \\ Full list of author information is \\ available at the end of the article
}

\begin{abstract}
In this paper, we consider the primitive three-dimensional viscous equations for large-scale atmosphere dynamics with topography effects and water vapor phase transition process. This modified climate model is commonly used in weather and climate predictions, and few theoretical analyses have been performed on them. The existence and uniqueness of a global strong solution to this climate model is established based on the initial data assumptions.
\end{abstract}

MSC: 35Q35; 76D03

Keywords: Climate dynamics; Strong solution; Moist atmospheric; Topography; Existence

\section{Introduction}

Applying the Boussinesq and hydrostatic approximation, the so-called primitive atmospheric equations can be derived, and this system is formulated in terms of the NavierStokes equations with the Coriolis force, the thermodynamic equations and the diffusion equation for vapor [19, 22, 28]. However, few studies have considered the effects of topography, changes of the external forcing with time and water vapor phase transitions, which have remarkable influences on climate dynamics. Based on realistic conditions, Zeng [29] showed the modified climate dynamics model in the following ways: (1) the effects of topography on the climate dynamics are considered; (2) the phase change of water vapor is studied; (3) the upper atmospheric pressure is set to zero; and (4) the anelastic approximation is not required in the system.

Then we show a moving frame $(\theta, \lambda, \zeta, t)$, where $\theta \in[0, \pi]$ is the colatitude, $\lambda \in[0,2 \pi]$ is the longitude, $\zeta=p / p_{s} \in[0,1], p \in\left[0, p_{s}\right]$ is the atmospheric pressure, where $p_{s}(\theta, \lambda, t)$ is the atmospheric pressure on the surface of the Earth, and $t$ is the time. The atmospheric state functions can be defined by the atmospheric horizontal velocity $V=\left(v_{\theta}, v_{\lambda}\right)$, vertical velocity $\dot{\zeta}$, temperature deviation $T^{\prime}$, geopotential deviation $\Phi^{\prime}$ and Earth surface pressure deviation $p_{s}^{\prime}$, the specific humidity $q$, and the liquid water content $m_{w}$. As the reference standard temperature $\widetilde{T}(\zeta)$, the reference standard geopotential $\widetilde{\Phi}(\zeta)$ and the reference standard Earth surface pressure $\widetilde{p}_{s}(\theta, \lambda, t)$ are determined, we can find that

(c) The Author(s) 2020. This article is licensed under a Creative Commons Attribution 4.0 International License, which permits use sharing, adaptation, distribution and reproduction in any medium or format, as long as you give appropriate credit to the original author(s) and the source, provide a link to the Creative Commons licence, and indicate if changes were made. The images or other third party material in this article are included in the article's Creative Commons licence, unless indicated otherwise in a credit line to the material. If material is not included in the article's Creative Commons licence and your intended use is not permitted by statutory regulation or exceeds the permitted use, you will need to obtain permission directly from the copyright holder. To view a copy of this licence, visit http://creativecommons.org/licenses/by/4.0/. 
$\widetilde{T}(\zeta)+T^{\prime}(\theta, \lambda, \zeta, t)$ is the atmospheric temperature $T(\theta, \lambda, \zeta, t), \widetilde{\Phi}(\zeta)+\Phi^{\prime}(\theta, \lambda, \zeta, t)$ is the geopotential $\Phi(\theta, \lambda, \zeta, t)$ and $\widetilde{p_{s}}(\theta, \lambda, t)+p_{s}^{\prime}(\theta, \lambda, t)$ is the surface pressure of Earth $p_{s}(\theta, \lambda, t)$. All of these state functions satisfy the following system:

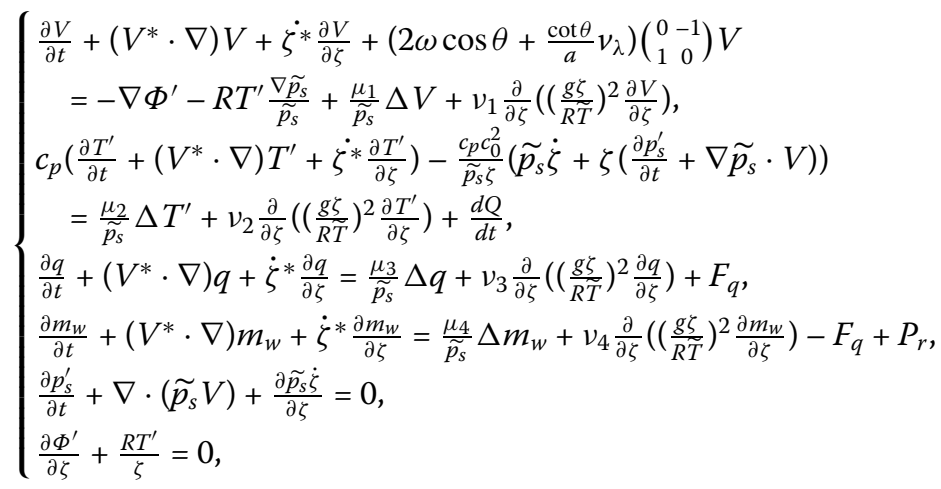

where $\omega$ is the Earth's rotation angular velocity; $g$ is the gravitational acceleration; $c_{0}, c_{p}$ and $R$ are thermodynamic parameters; $\mu_{i}$ and $v_{i}(i=1,2,3,4)$ are the diffusion coefficients; $2 \omega \cos \theta\left(\begin{array}{cc}0 & -1 \\ 1 & 0\end{array}\right) V$ stands for the Coriolis force on the atmosphere.

Moreover, we introduce $d Q / d t$, which is composed of the radiant heating $H_{1}$ and the latent heating $\mathrm{H}_{2}$ caused by the water vapor phase transition, and they give the forms

$$
\frac{d Q}{d t}=H_{1}+H_{2}
$$

Here, we simply assume that Newtonian cooling holds,

$$
H_{1}=-\kappa_{a} T^{\prime},
$$

where $\kappa_{a}$ is a positive constant.

$\mathrm{H}_{2}$ should be closely related to the microphysics processes of condensation and evaporation, and we have

$$
H_{2}=-L F_{q} \text {, }
$$

where $L$ is the latent heat constant, and

$$
F_{q}=\delta_{21} \delta_{22}\left(L \dot{\zeta} \frac{W(T)}{\zeta}\right)
$$

which represents the mass of water that is added by condensation or removed by evaporation. $\delta_{21}$ and $\delta_{22}$ have the following forms:

$$
\left\{\begin{array}{l}
\delta_{21}=\delta_{1}\left(q-q_{m}\right)= \begin{cases}1, & q>q_{m}, \\
0, & q \leq q_{m},\end{cases} \\
\delta_{22}=\delta_{2}\left(m_{w}\right)= \begin{cases}1, & m_{w}>0, \\
0, & m_{w} \leq 0,\end{cases}
\end{array}\right.
$$


where $q_{m}$ is the saturation special humidity. We assume that $W(T)$ is a globally Lipschitz bounded function, namely

$$
W(T)=q_{m} T\left(\frac{R L-c_{p} R_{v} T}{c_{p} R_{v} T^{2}+L^{2} q_{m}}\right)
$$

where $R_{v}$ is the gas constant for water vapor. The term $P_{r}$ is the precipitation rate, which takes the following form:

$$
P_{r}=h_{1}\left(F_{q}\right)=h_{1}\left(\delta_{21} \delta_{22}\left(L \dot{\zeta} \frac{W(T)}{\zeta}\right)\right),
$$

where

$$
h_{1}(x)= \begin{cases}\alpha x-\beta, & x<0, \\ 0, & x>0,\end{cases}
$$

and $0<\alpha<1, \beta>0$.

In this work, we show that the differential operators $\operatorname{grad}:=\nabla \operatorname{div}:=\nabla \cdot$ and $\Delta$ on the spherical surface are

$$
\left\{\begin{array}{l}
\nabla=\left(\frac{1}{a} \frac{\partial}{\partial \theta}, \frac{1}{a \sin \theta} \frac{\partial}{\partial \lambda}\right) \\
\nabla \cdot V=\frac{1}{a \sin \theta} \frac{\partial\left(\sin \theta v_{\theta}\right)}{\partial \theta}+\frac{1}{a \sin \theta} \frac{\partial \nu_{\lambda}}{\partial \lambda} \\
\Delta=\frac{1}{a^{2} \sin \theta} \frac{\partial}{\partial \theta}\left(\sin \theta \frac{\partial}{\partial \theta}\right)+\frac{1}{a^{2} \sin ^{2} \theta} \frac{\partial^{2}}{\partial \lambda^{2}}
\end{array}\right.
$$

where $a$ is Earth radius. And we study the system on $\Omega \times[0, M]:=S^{2} \times[0,1] \times[0, M]=$ $[0, \pi] \times[0,2 \pi] \times[0,1] \times[0, M]$, where $M>0$ is some time.

Moveover, we choose the modified smooth velocity field $\left(V^{*}, \zeta^{*}\right)[18]$. Here we set $\bar{V}:=$ $\int_{0}^{1} V(\theta, \lambda, \zeta, t) d \zeta$, and decompose $\widetilde{p}_{s} \bar{V}$ into the three parts via

$$
\tilde{p}_{s} \bar{V}=\nabla(\chi-\Psi)+\nabla \Psi+\left(\begin{array}{cc}
0 & -1 \\
1 & 0
\end{array}\right) \nabla \psi,
$$

where the function $\Psi$ satisfies

$$
\Delta \Psi=-\frac{\partial \widetilde{p_{s}}}{\partial t},
$$

and $V^{*}$ can be obtained as follows:

$$
V^{*}=V-\widetilde{p}_{s}^{-1} \nabla(\chi-\Psi)=\left(v_{\theta}^{*}, v_{\lambda}^{*}\right) .
$$

Meanwhile, we get $\dot{\zeta}^{*}$ as the solution of

$$
\frac{\partial \widetilde{p_{s}}}{\partial t}+\frac{1}{a \sin \theta}\left(\frac{\partial \widetilde{p_{s}} v_{\theta}^{*} \sin \theta}{\partial \theta}+\frac{\partial \widetilde{p_{s}} v_{\lambda}^{*}}{\partial \lambda}\right)+\frac{\partial \widetilde{p_{s}} \dot{\zeta}^{*}}{\partial \zeta}=0
$$

with the boundary condition

$$
\dot{\zeta}^{*}=0, \quad \text { as } \zeta=0
$$


which implies that

$$
\begin{aligned}
\dot{\zeta}^{*} & =-\frac{1}{\widetilde{p}_{s}} \int_{0}^{\zeta}\left(\frac{1}{a \sin \theta}\left(\frac{\partial \widetilde{p}_{s} v_{\theta}^{*} \sin \theta}{\partial \theta}+\frac{\partial \widetilde{p}_{s} v_{\lambda}^{*}}{\partial \lambda}\right)+\frac{\partial \widetilde{p_{s}}}{\partial t}\right) d s \\
& =-\frac{1}{\widetilde{p_{s}}} \int_{0}^{\zeta} \nabla \cdot\left(\widetilde{p}_{s} V^{*}\right) d s-\frac{1}{\widetilde{p_{s}}} \frac{\partial \widetilde{p_{s}}}{\partial t} \zeta .
\end{aligned}
$$

Then from the definition of $\bar{V}$, we know

$$
\int_{0}^{1} \frac{1}{a \sin \theta}\left(\frac{\partial \widetilde{p}_{s} v_{\theta}^{*} \sin \theta}{\partial \theta}+\frac{\partial \widetilde{p_{s}} v_{\lambda}^{*}}{\partial \lambda}\right) d \zeta=\int_{0}^{1} \nabla \cdot\left(\widetilde{p}_{s} V^{*}\right) d \zeta=-\frac{\partial \widetilde{p_{s}}}{\partial t}
$$

and we have

$$
\dot{\zeta}^{*}=0, \quad \text { as } \zeta=1
$$

Then we can give the boundary conditions without relief are as follows: All functions are $\pi$ periodical with respect to $\theta, 2 \pi$ periodical with respect to $\lambda$, and

$$
\left\{\begin{array}{l}
\left.\frac{\partial V}{\partial \zeta}\right|_{\zeta=0}=\left.\frac{\partial T^{\prime}}{\partial \zeta}\right|_{\zeta=0}=\left.\frac{\partial q}{\partial \zeta}\right|_{\zeta=0}=\left.\frac{\partial m_{w}}{\partial \zeta}\right|_{\zeta=0}=\left.\dot{\zeta}\right|_{\zeta=0}=0, \\
\left.\left(v_{1} \frac{\partial V}{\partial \zeta}+k_{s 1} f(|V|) V\right)\right|_{\zeta=1}=0,\left.\quad\left(v_{2} \frac{\partial T^{\prime}}{\partial \zeta}+k_{s 2} T^{\prime}\right)\right|_{\zeta=1}=0, \\
\left.\left(v_{3} \frac{\partial q}{\partial \zeta}+k_{s 3} f\left(\left|V_{10}\right|\right)\left(q-q_{m}^{*}\right)\right)\right|_{\zeta=1}=0,\left.\quad \frac{\partial m_{w}}{\partial \zeta}\right|_{\zeta=1}=0, \\
\left.\dot{\zeta}\right|_{\zeta=1}=0,\left.\quad \Phi^{\prime}\right|_{\zeta=1}=\frac{R \widetilde{T}_{s}}{\widetilde{p}_{s}} p_{s}^{\prime}(\theta, \lambda, t),
\end{array}\right.
$$

where the given function $\widetilde{T}_{s}(\theta, \lambda)$ is the reference standard surface temperature of Earth, $q_{m}^{*}$ is the reference standard surface saturation special humidity of Earth, the given function $V_{10}(\theta, \lambda)$ is the 10 -m wind speed, $k_{s 1}, k_{s 2}$ and $k_{s 3}$ are positive constants and the chilling coefficient $f(|V|)$ is a positive function of $|V|$.

There is a huge literature on the study of various atmospheric problems; e.g., in the 1990s, Lions et al. [19-21] gave the new formulation for the primitive equations of largescale atmosphere and ocean, and proved the existence of global weak solutions to the initial boundary value problem. The existence of global attractors to the primitive equations associated with the atmospheric evolution process have been studied by Chepzhov and Vishik [9]. Wu et al. [24] obtained the existence of global weak solutions to the climate model with the effects of topography. Furthermore, Huang and Guo $[14,15]$ proved the existence of the atmospheric global attractors of the atmospheric motion model without or with the effects of topography, respectively, and they also obtained the existence and the asymptotic behaviors of a weak solution. Recently, Lian et al. $[16,18]$ addressed the $L^{1}$-stability of weak solutions to the atmospheric equations with or without the effects of topography.

Meanwhile, there are many studies of strong solution for viscous primitive large-scale ocean and atmosphere equations. Taking the values of the initial data to be sufficiently small, the global existence of a strong solution to primitive equations was investigated by Guillén-González et al. [10], and the local existence of strong solution to the system for all initial data was also proved. Furthermore, Temam and Ziane [23] considered the coupled atmosphere-ocean equations and showed the local existence of strong solution. Cao 
and Titi [5] proved the global well-posedness and finite-dimensional global attractors of the 3D planetary geostrophic model. The well-posedness and long-time behavior of the strong solution to the horizontal hyper-diffusion 3D thermocline planetary geostrophic model were also obtained by Cao et al. [8]. In particular, for all initial data, Cao and Titi [6] proved the global existence of strong solution to three-dimensional viscous primitive equations. Furthermore, Cao et al. [1, 7] investigated the global well-posedness of threedimensional viscous primitive equations with only vertical diffusion, and proved the global existence of strong solution using $H^{2}$ initial data. Cao et al. [2] also considered the initial boundary value problem of the primitive equations with only horizontal diffusion in the temperature equation, and they obtained the global existence of strong solutions using $H^{2}$ initial data. Recently, the initial boundary value problem of the primitive equations with only horizontal eddy viscosity in the horizontal momentum equations and only horizontal diffusion in the temperature equation were addressed by Cao et al. [3]. Cao et al. [4] also studied the 3D primitive equations with only horizontal eddy viscosity in the horizontal momentum equations and only vertical diffusivity in the temperature equation. In these studies, Cao et al. gave the $H^{2}$ regularity estimates of the strong solution. However, the upper atmospheric pressure in the references above is treated as a positive constant, in particular, Lian and Zeng[17] proved the existence of strong solution and trajectory attractor to a modified climate dynamic model, while the upper atmospheric pressure is treated as zero.

Up to now, there are some important studies about the well-posedness of the moist atmosphere. For example, Guo and Huang [11] proved the existence of global weak solutions and attractors to the primitive equations of a moist atmosphere. In addition, Guo and Huang [12] investigated the existence and uniqueness of global strong solution, and they addressed the existence of the universal attractor for the large-scale moist atmosphere. Under a physically reasonable assumption, Zelati and Temam [27] derived the existence and uniqueness of solution for the specific humidity equation. They also studied the coupling of the specific humidity equation with the temperature equation. Based on [27], Zelati et al. [25] addressed the uniqueness of solution for the system of moist atmosphere with saturation. Additional properties and regularity results of the solutions were also proven. Zelati et al. [26] proved the global existence of quasi-strong and strong solutions of primitive equations in the interest of thinking over vital phase transition phenomena due to air saturation and condensation. Using the ideas of Cao and Titi [6], Hittmeir et al. [13] showed the well-posedness for the full moist atmospheric flow model, where the moisture model is coupled to the large-scale atmosphere equations.

In this paper, we investigate the initial boundary value problem for the climate dynamics model with effects of topography and water vapor phase transition. Moreover, the heating is caused by internal sources depending on the atmospheric motion rather than by an external one, and the internal heating function due to the phase change of water vapor is approximated by some properly analytical functions suitable for mathematical analyses. Meanwhile, the upper atmospheric pressure is treated as zero. Based on the initial data assumptions, the existence and uniqueness of a global strong solution to this modified climate model is established.

We organize this paper as follows. In Sect. 2, we present the major results associated with the existence of a unique global strong solution. Section 3 gives some useful lem- 
mas. In Sect. 4, we will provide some useful proofs, followed by several important a priori estimates. Finally, the conclusions are drawn in Sect. 5.

\section{Main results}

We will show the global well-posedness of strong solution to (1.1) and give a simple version of system (1.1) firstly. From the boundary conditions (1.19), we have

$$
\begin{aligned}
& p_{s}^{\prime}(\theta, \lambda, t)=-\int_{0}^{t} \nabla \cdot\left(\widetilde{p}_{s}(\theta, \lambda) \bar{V}(\theta, \lambda, \tau)\right) d \tau, \\
& \widetilde{p}_{s} \dot{\zeta}=-\frac{\partial p_{s}^{\prime}}{\partial t} \zeta-\int_{0}^{\zeta} \nabla \cdot\left(\widetilde{p}_{s} V\right) d s=\nabla \cdot\left(\widetilde{p}_{s} \bar{V}\right) \zeta-\int_{0}^{\zeta} \nabla \cdot\left(\widetilde{p}_{s} V\right) d s,
\end{aligned}
$$

and

$$
\Phi^{\prime}=\frac{R \widetilde{T}_{s}}{\widetilde{p}_{s}} p_{s}^{\prime}(\theta, \lambda, t)+R \int_{\zeta}^{1} \frac{T^{\prime}(\theta, \lambda, s, t)}{s} d s
$$

Substitute (2.1)-(2.3) into (1.1) and define the unknown function $U:=\left(V, T^{\prime}, q, m_{w}\right)^{\mathrm{T}}$, then we give the simplification of the system (1.1)

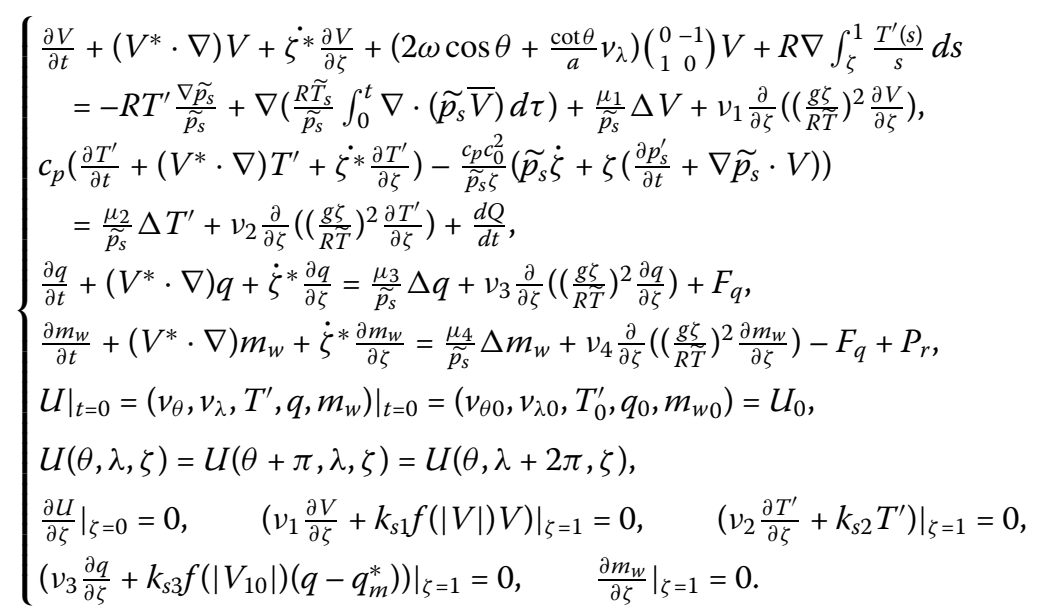

Then we can state the main results of the present paper as follows.

Theorem 2.1 For any $M>0$, we assume that

$$
\begin{aligned}
& \widetilde{T}(\zeta) \in C^{1}(0,1), \quad \widetilde{T}(\zeta) \geq 0, \quad \widetilde{T}^{\prime}(\zeta) \geq 0, \quad \lim _{\zeta \rightarrow 0} \frac{\zeta}{\widetilde{T}(\zeta)}:=T_{0}>0, \\
& \widetilde{T}_{s}(\theta, \lambda), \quad \widetilde{p}_{s}(\theta, \lambda, t), \\
& \quad \tilde{p}_{s}^{-1}(\theta, \lambda, t) \in W^{1, \infty}\left([0, M] ; W^{1, \infty}([0, \pi] \times[0,2 \pi])\right), \\
& V_{10}(\theta, \lambda), \quad V_{10}^{-1}(\theta, \lambda) \in L^{\infty}([0, \pi] \times[0,2 \pi]), \\
& q_{m}(\theta, \lambda) \in L^{\infty}([0, \pi] \times[0,2 \pi]), \quad q_{m}^{*}(\theta, \lambda) \in W^{1, \infty}([0, \pi] \times[0,2 \pi]), \\
& f(s) \in C\left(\mathbb{R}^{+}\right), \quad C_{1} s^{\alpha} \leq f(s) \leq C_{2}\left(1+s^{\alpha}\right), \quad 0 \leq \alpha<1, \\
& \left|W\left(s_{1}\right)-W\left(s_{2}\right)\right| \leq C_{3}\left|s_{1}-s_{2}\right|, \quad \forall s_{1}, s_{2} \in R,
\end{aligned}
$$




$$
|W(s)| \leq C_{4}, \quad \forall s \in R,
$$

where $W(s)$ is a globally Lipschitz bounded function, $T_{0}, C_{1}, C_{2}, C_{3}$ and $C_{4}$ are positive constants.

Let $U_{0}=\left(V_{0}, T_{0}^{\prime}, q_{0}, m_{w 0}\right) \in H^{1}(\Omega)$, then there exists a unique global strong solution $U$ to the system (2.4) on the interval $[0, M]$ satisfying

$$
\left\{\begin{array}{l}
V \in C\left([0, M] ; H^{1}(\Omega)\right) \cap L^{2}\left(0, M ; H^{2}(\Omega)\right), \\
T \in C\left([0, M] ; H^{1}(\Omega)\right) \cap L^{2}\left(0, M ; H^{2}(\Omega)\right), \\
q \in C\left([0, M] ; H^{1}(\Omega)\right) \cap L^{2}\left(0, M ; H^{2}(\Omega)\right), \\
m_{w} \in C\left([0, M] ; H^{1}(\Omega)\right) \cap L^{2}\left(0, M ; H^{2}(\Omega)\right) .
\end{array}\right.
$$

\section{Some lemmas}

Lemma 3.1 Note that, from [17], letting $\phi \in C^{\infty}\left(S^{2}\right)$ and $V, V_{1} \in C^{\infty}\left(S^{2}\right)$, we have

$$
\int_{S^{2}} \nabla \phi \cdot V d \sigma=-\int_{S^{2}} \phi \nabla \cdot V d \sigma
$$

and

$$
-\int_{\Omega} \Delta V \cdot V_{1} d \sigma d \zeta=\int_{\Omega} \frac{\partial V}{\partial \theta} \cdot \frac{\partial V_{1}}{\partial \theta} d \sigma d \zeta+\int_{\Omega} \frac{\partial V}{\partial \lambda} \cdot \frac{\partial V_{1}}{\partial \lambda} d \sigma d \zeta
$$

Lemma 3.2 Let $V, T, q, m_{w} \in H^{1}(\Omega)$, then for $n=1,2,3$

$$
\begin{aligned}
& \int_{\Omega}\left(\widetilde{p_{s}} V^{*} \cdot \nabla V-\left(\int_{0}^{\zeta} \nabla \cdot\left(\widetilde{p_{s}} V^{*}\right)+\zeta \frac{\partial \widetilde{p_{s}}}{\partial t}\right) \frac{\partial V}{\partial \zeta}\right) \cdot V d \sigma d \zeta=0, \\
& \int_{\Omega}\left(\widetilde{p_{s}} V^{*} \cdot \nabla T^{\prime}-\left(\int_{0}^{\zeta} \nabla \cdot\left(\widetilde{p_{s}} V^{*}\right)+\zeta \frac{\partial \widetilde{p_{s}}}{\partial t}\right) \frac{\partial T^{\prime}}{\partial \zeta}\right) T^{\prime n} d \sigma d \zeta=0, \\
& \int_{\Omega}\left(\widetilde{p_{s}} V^{*} \cdot \nabla q-\left(\int_{0}^{\zeta} \nabla \cdot\left(\widetilde{p_{s}} V^{*}\right)+\zeta \frac{\partial \widetilde{p_{s}}}{\partial t}\right) \frac{\partial q}{\partial \zeta}\right) q^{n} d \sigma d \zeta=0, \\
& \int_{\Omega}\left(\tilde{p_{s}} V^{*} \cdot \nabla m_{w}-\left(\int_{0}^{\zeta} \nabla \cdot\left(\widetilde{p_{s}} V^{*}\right)+\zeta \frac{\partial \widetilde{p_{s}}}{\partial t}\right) \frac{\partial m_{w}}{\partial \zeta}\right) m_{w}^{n} d \sigma d \zeta=0,
\end{aligned}
$$

and

$$
\int_{\Omega}\left(\int_{\zeta}^{1} \frac{\nabla T^{\prime}(s)}{s} d s \cdot\left(\widetilde{p_{s}} V\right)+\frac{1}{\zeta}\left(\int_{0}^{\zeta} \nabla \cdot\left(\widetilde{p_{s}} V\right) d s\right) T^{\prime}\right) d \sigma d \zeta=0 .
$$

Furthermore, we recall some useful interpolation inequalities as follows:

(1) For $f \in H^{1}\left(S^{2}\right)$,

$$
\begin{aligned}
& \|f\|_{L^{4}\left(S^{2}\right)} \leq C\|f\|_{L^{2}\left(S^{2}\right)}^{\frac{1}{2}}\|f\|_{H^{1}\left(S^{2}\right)}^{\frac{1}{2}}, \\
& \|f\|_{L^{6}\left(S^{2}\right)} \leq C\|f\|_{L^{4}\left(S^{2}\right)}^{\frac{2}{3}}\|f\|_{H^{1}\left(S^{2}\right)^{\prime}}^{\frac{1}{3}}, \\
& \|f\|_{L^{8}\left(S^{2}\right)} \leq C\|f\|_{L^{4}\left(S^{2}\right)}^{\frac{1}{2}}\|f\|_{H^{1}\left(S^{2}\right)}^{\frac{1}{2}} .
\end{aligned}
$$


(2) For $f \in H^{1}(\Omega)$,

$$
\|f\|_{L^{4}(\Omega)} \leq C\|f\|_{L^{2}(\Omega)}^{\frac{1}{4}}\|f\|_{H^{1}(\Omega)}^{\frac{3}{4}} .
$$

\section{The a priori estimates}

Next, we will consider the a priori estimates for the strong solution $U$ to the system (2.4). Using definition of the fluctuation $\widetilde{V}$ in [17], we can give the equations of the fluctuation $\widetilde{V}$ as follows:

$$
\begin{aligned}
\frac{\partial \bar{V}}{\partial t}+ & \int_{0}^{1}\left(\nabla_{V^{*}} V-\left(\frac{1}{\widetilde{p}_{s}} \int_{0}^{\zeta} \nabla \cdot\left(\widetilde{p_{s}} V^{*}\right)+\zeta \frac{\partial \widetilde{p}_{s}}{\partial t}\right) \frac{\partial V}{\partial \zeta}\right) d \zeta+2 \omega \cos \theta\left(\begin{array}{cc}
0 & -1 \\
1 & 0
\end{array}\right) \bar{V} \\
& +R \int_{0}^{1} \int_{\zeta}^{1} \frac{\nabla T^{\prime}(s)}{s} d s d \zeta+R \frac{\nabla \widetilde{p_{s}}}{\widetilde{p_{s}}} \int_{0}^{1} T^{\prime} d \zeta-\nabla\left(\frac{R \widetilde{T}_{s}}{\widetilde{p_{s}}} \int_{0}^{t} \nabla \cdot\left(\widetilde{p_{s}} \bar{V}\right) d \tau\right) \\
= & \frac{\mu_{1}}{\widetilde{p_{s}}} \Delta \bar{V}-\left.k_{s 1}\left(\left(\frac{g \zeta}{R \widetilde{T}}\right)^{2} f(|V|) V\right)\right|_{\zeta=1}
\end{aligned}
$$

where

$$
\nabla_{V^{*}} V=\frac{1}{a}\left(a^{2} V^{*} \cdot \nabla V^{*}+v_{\lambda} \cot \theta\left(\begin{array}{cc}
0 & -1 \\
1 & 0
\end{array}\right) V\right) .
$$

We let

$$
\widetilde{V}=V-\bar{V}
$$

which also implies

$$
\widetilde{V}^{*}=V^{*}-\overline{V^{*}}=V-\bar{V}=\widetilde{V}, \quad \overline{V^{*}}=\bar{V}^{*} .
$$

Note that

$$
\widetilde{\widetilde{V}}=0, \overline{\widetilde{V}^{*}}=0, \quad \nabla \cdot\left(\widetilde{p}_{s} \overline{V^{*}}\right)=-\frac{\partial \widetilde{p_{s}}}{\partial t},
$$

and we have

$$
\begin{aligned}
& -\int_{0}^{1}\left(\int_{0}^{\zeta} \nabla \cdot\left(\widetilde{p}_{s} V^{*}\right) d s\right) \frac{\partial V}{\partial \zeta} d \zeta \\
& =\left.\frac{\partial \widetilde{p}_{s}}{\partial t} V\right|_{\zeta=1}+\int_{0}^{1} V \nabla \cdot\left(\widetilde{p}_{s} V^{*}\right) d \zeta \\
& \quad=\left.\frac{\partial \widetilde{p}_{s}}{\partial t} V\right|_{\zeta=1}-\frac{\partial \widetilde{p_{s}}}{\partial t} \bar{V}+\int_{0}^{1} \widetilde{V} \nabla \cdot\left(\widetilde{p_{s}} \widetilde{V}^{*}\right) d \zeta, \\
& -\frac{\partial \widetilde{p_{s}}}{\partial t} \int_{0}^{1} \zeta \frac{\partial V}{\partial \zeta} d \zeta=-\left.\frac{\partial \widetilde{p}_{s}}{\partial t} V\right|_{\zeta=1}+\frac{\partial \widetilde{p}_{s}}{\partial t} \bar{V},
\end{aligned}
$$

and

$$
\int_{0}^{1} \nabla_{V^{*}} V d \zeta=\int_{0}^{1} \nabla_{\widetilde{V}} \widetilde{V} d \zeta+\nabla_{\bar{V}^{*}} \bar{V},
$$


where

$$
\begin{aligned}
& \nabla_{\widetilde{V}^{*}} \widetilde{V}=\frac{1}{a}\left(a^{2} \widetilde{V}^{*} \cdot \nabla \widetilde{V}^{*}+\widetilde{v}_{\lambda} \cot \theta\left(\begin{array}{cc}
0 & -1 \\
1 & 0
\end{array}\right) \widetilde{V}\right), \\
& \nabla_{\bar{V}^{*}} \bar{V}=\frac{1}{a}\left(a^{2} \bar{V}^{*} \cdot \nabla \bar{V}^{*}+\bar{v}_{\lambda} \cot \theta\left(\begin{array}{cc}
0 & -1 \\
1 & 0
\end{array}\right) \bar{V}\right) .
\end{aligned}
$$

From (4.1), (4.6) and (4.7), we get

$$
\begin{aligned}
& \frac{\partial \bar{V}}{\partial t}+\nabla_{\overline{V^{*}}} \bar{V}+\overline{\frac{1}{\widetilde{p}_{s}} \widetilde{V} \nabla \cdot\left(\widetilde{p}_{s} \widetilde{V}^{*}\right)+\nabla_{\widetilde{V}^{*}} \widetilde{V}}+2 \omega \cos \theta\left(\begin{array}{cc}
0 & -1 \\
1 & 0
\end{array}\right) \bar{V} \\
& +R \int_{0}^{1} \int_{\zeta}^{1} \frac{\nabla T^{\prime}(s)}{s} d s d \zeta+R \frac{\nabla \widetilde{p_{s}}}{\widetilde{p}_{s}} \int_{0}^{1} T^{\prime} d \zeta-\nabla\left(\frac{R \widetilde{T}_{s}}{\widetilde{p_{s}}} \int_{0}^{t} \nabla \cdot\left(\widetilde{p_{s}} \bar{V}\right) d \tau\right) \\
& =\frac{\mu_{1}}{\widetilde{p_{s}}} \Delta \bar{V}-\left.k_{s 1}\left(\left(\frac{g \zeta}{R \widetilde{T}}\right)^{2} f(|V|) V\right)\right|_{\zeta=1} .
\end{aligned}
$$

Subtracting (4.11) from $(2.4)_{1}$, we also find that the fluctuation $\widetilde{V}$ satisfies the following equation:

$$
\begin{aligned}
& \frac{\partial \widetilde{V}}{\partial t}+\nabla_{\widetilde{V}^{*}} \widetilde{V}-\left(\frac{1}{\widetilde{p_{s}}} \int_{0}^{\zeta} \nabla \cdot\left(\widetilde{p}_{s} \widetilde{V}^{*}\right) d s\right) \frac{\partial \widetilde{V}}{\partial \zeta}+\nabla_{\bar{V}^{*}} \widetilde{V}+\nabla_{\widetilde{V}^{*}} \bar{V} \\
& -\overline{\frac{1}{\widetilde{p}_{s}} \widetilde{V} \nabla \cdot\left(\widetilde{p}_{s} \widetilde{V}^{*}\right)+\nabla_{\widetilde{V}^{*}} \widetilde{V}}+2 \omega \cos \theta\left(\begin{array}{cc}
0 & -1 \\
1 & 0
\end{array}\right) \widetilde{V}+R \int_{\zeta}^{1} \frac{\nabla T^{\prime}(s)}{s} d s \\
& -R \int_{0}^{1} \int_{\zeta}^{1} \frac{\nabla T^{\prime}(s)}{s} d s d \zeta+R \frac{\nabla \widetilde{p_{s}}}{\widetilde{p_{s}}} T^{\prime}-R \frac{\nabla \widetilde{p_{s}}}{\widetilde{p_{s}}} \int_{0}^{1} T^{\prime} d \zeta \\
& -\left.k_{s 1}\left(\left(\frac{g \zeta}{R \widetilde{T}}\right)^{2} f(|V|) V\right)\right|_{\zeta=1}=\frac{\mu_{1}}{\widetilde{p_{s}}} \Delta \widetilde{V}+v_{1} \frac{\partial}{\partial \zeta}\left(\left(\frac{g \zeta}{R \widetilde{T}}\right)^{2} \frac{\partial \widetilde{V}}{\partial \zeta}\right),
\end{aligned}
$$

with the boundary conditions

$$
\left.\frac{\partial \widetilde{V}}{\partial \zeta}\right|_{\zeta=0}=0,\left.\quad\left(v_{1} \frac{\partial \widetilde{V}}{\partial \zeta}+k_{s 1} f(|V|) V\right)\right|_{\zeta=1}=0
$$

where

$$
\begin{aligned}
& \nabla_{\bar{V}^{*}} \tilde{V}=\frac{1}{a}\left(a^{2} \bar{V}^{*} \cdot \nabla \widetilde{V}^{*}+\bar{v}_{\lambda} \cot \theta\left(\begin{array}{cc}
0 & -1 \\
1 & 0
\end{array}\right) \widetilde{V}\right), \\
& \nabla_{\widetilde{V}^{*}} \bar{V}=\frac{1}{a}\left(a^{2} \widetilde{V}^{*} \cdot \nabla \bar{V}^{*}+\widetilde{v}_{\lambda} \cot \theta\left(\begin{array}{cc}
0 & -1 \\
1 & 0
\end{array}\right) \bar{V}\right) .
\end{aligned}
$$

Then we have the usual energy inequality as follows. 
Lemma 4.1 Under the assumptions of Theorem 2.1, for any $M>0$ given, the strong solution $U$ to the system (2.4) satisfies

$$
\begin{aligned}
& \|V\|_{L^{2}(\Omega)}^{2}+\left\|T^{\prime}\right\|_{L^{2}(\Omega)}^{2}+\|q\|_{L^{2}(\Omega)}^{2}+\left\|m_{w}\right\|_{L^{2}(\Omega)}^{2}+\left\|\int_{0}^{t} \nabla \cdot\left(\widetilde{p}_{s} \bar{V}\right) d \tau\right\|_{L^{2}(\Omega)}^{2} \\
& \quad+\int_{0}^{t}\|U\|_{H^{1}(\Omega)}^{2} d \tau+\int_{0}^{t}\left\|T^{\prime}\right\|_{L^{2}(\Omega)}^{2} d \tau+\left.\int_{0}^{t} \int_{S^{2}} f(|V|)|V|^{2}\right|_{\zeta=1} d \sigma d \tau \\
& \quad+\left.\int_{0}^{t} \int_{S^{2}} T^{\prime 2}\right|_{\zeta=1} d \sigma d \tau+\left.\int_{0}^{t} \int_{S^{2}} f\left(\left|V_{10}\right|\right) q^{2}\right|_{\zeta=1} d \sigma d \tau \leq C(M), \quad t \in[0, M]
\end{aligned}
$$

where $C(M)>0$ denotes a constant dependent of time $M$.

Proof Multiplying (2.4) by $\widetilde{p}_{s} U$ and using the boundary conditions, we obtain

$$
\begin{aligned}
\frac{1}{2} \frac{d}{d t} & \int_{\Omega} \widetilde{p}_{s}\left(V^{2}+\frac{R}{c_{0}^{2}} T^{2}+q^{2}+m_{w}^{2}\right) d \sigma d \zeta+\frac{d}{d t} \int_{S^{2}} \frac{R \widetilde{T}_{s}}{\widetilde{p}_{s}}\left(\int_{0}^{t} \nabla \cdot\left(\widetilde{p}_{s} \bar{V}\right) d \tau\right)^{2} d \sigma \\
& +\mu_{1} \int_{\Omega}|\nabla V|^{2} d \sigma d \zeta+\frac{\mu_{2} R}{c_{p} c_{0}^{2}} \int_{\Omega}\left|\nabla T^{\prime}\right|^{2} d \sigma d \zeta \\
& +\mu_{3} \int_{\Omega}|\nabla q|^{2} d \sigma d \zeta+\mu_{4} \int_{\Omega}\left|\nabla m_{w}\right|^{2} d \sigma d \zeta \\
& +v_{1} \int_{\Omega} \widetilde{p}_{s}\left(\frac{g \zeta}{R \widetilde{T}}\right)^{2}\left(\frac{\partial V}{\partial \zeta}\right)^{2} d \sigma d \zeta+\frac{v_{2} R}{c_{p} c_{0}^{2}} \int_{\Omega} \widetilde{p}_{s}\left(\frac{g \zeta}{R \widetilde{T}}\right)^{2}\left(\frac{\partial T^{\prime}}{\partial \zeta}\right)^{2} d \sigma d \zeta \\
& +v_{3} \int_{\Omega} \widetilde{p}_{s}\left(\frac{g \zeta}{R \widetilde{T}}\right)^{2}\left(\frac{\partial q}{\partial \zeta}\right)^{2} d \sigma d \zeta+v_{4} \int_{\Omega} \widetilde{p}_{s}\left(\frac{g \zeta}{R \widetilde{T}}\right)^{2}\left(\frac{\partial m_{w}}{\partial \zeta}\right)^{2} d \sigma d \zeta \\
& +\left.k_{s 1} \int_{S^{2}} \widetilde{p}_{s}\left(\frac{g \zeta}{R \widetilde{T}}\right)^{2} f(|V|)|V|^{2}\right|_{\zeta=1} d \sigma+\left.\frac{k_{s 2} R}{c_{p} c_{0}^{2}} \int_{S^{2}} \widetilde{p}_{s}\left(\frac{g \zeta}{R \widetilde{T}}\right)^{2} T^{\prime 2}\right|_{\zeta=1} d \sigma \\
& +\left.k_{s 3} \int_{S^{2}} f\left(\left|V_{10}\right|\right)\left(\frac{g \zeta}{R \widetilde{T}}\right)^{2}\left(q-q_{m}^{*}\right) q\right|_{\zeta=1} d \sigma+\int_{\Omega} \frac{R \widetilde{p}_{s}}{c_{p} c_{0}^{2}} \kappa_{a} T^{\prime 2} d \sigma d \zeta \\
= & \frac{1}{2} \int_{\Omega} \frac{d \widetilde{p}_{s}}{d t}\left(V^{2}+\frac{R}{c_{0}^{2}} T^{\prime 2}+q^{2}+m_{w}^{2}\right) d \sigma d \zeta-\int_{\Omega} \frac{R \widetilde{p}_{s}}{c_{p} c_{0}^{2}} \delta_{21} \delta_{22} \dot{\zeta} \frac{W(T)}{\zeta} T^{\prime} d \sigma d \zeta \\
& +\int_{\Omega} \widetilde{p}_{s} q \delta_{21} \delta_{22} \dot{\zeta} \frac{W(T)}{\zeta} d \sigma d \zeta-\int_{\Omega} \widetilde{p}_{s} m_{w} \delta_{21} \delta_{22} \dot{\zeta} \frac{W(T)}{\zeta} d \sigma d \zeta \\
& +\int_{\Omega} \widetilde{p}_{s} m_{w} h_{1}\left(\delta_{21} \delta_{22} \dot{\zeta} \frac{W(T)}{\zeta}\right) d \sigma d \zeta .
\end{aligned}
$$

Thanks to the Young inequality, the Hardy inequality and the Hölder inequality, we get

$$
\begin{aligned}
& \left|\int_{\Omega} \delta_{21} \delta_{22} \dot{\zeta} \frac{W(T)}{\zeta} T^{\prime} d \sigma d \zeta\right| \\
& \quad \leq C \int_{\Omega} T^{\prime 2} d \sigma d \zeta+\varepsilon \int_{\Omega}\left|\nabla \cdot\left(\widetilde{p}_{s} \bar{V}\right)\right|^{2} d \sigma d \zeta+\varepsilon \int_{\Omega}\left(\frac{1}{\zeta} \int_{0}^{\zeta} \nabla \cdot\left(\widetilde{p}_{s} V\right) d s\right)^{2} d \sigma d \zeta \\
& \quad \leq C \int_{\Omega} T^{\prime 2} d \sigma d \zeta+C \int_{\Omega}|V|^{2} d \sigma d \zeta+\varepsilon C \int_{\Omega}|\nabla V|^{2} d \sigma d \zeta \\
& \left|\int_{\Omega} \widetilde{p}_{s} q \delta_{21} \delta_{22} \dot{\zeta} \frac{W(T)}{\zeta} d \sigma d \zeta\right|
\end{aligned}
$$




$$
\begin{aligned}
& \quad \leq C \int_{\Omega} q^{2} d \sigma d \zeta+\varepsilon \int_{\Omega}\left|\nabla \cdot\left(\widetilde{p}_{s} \bar{V}\right)\right|^{2} d \sigma d \zeta+\varepsilon \int_{\Omega}\left(\frac{1}{\zeta} \int_{0}^{\zeta} \nabla \cdot\left(\widetilde{p}_{s} V\right) d s\right)^{2} d \sigma d \zeta \\
& \quad \leq C \int_{\Omega} q^{2} d \sigma d \zeta+C \int_{\Omega}|V|^{2} d \sigma d \zeta+\varepsilon C \int_{\Omega}|\nabla V|^{2} d \sigma d \zeta \\
& \left|\int_{\Omega} \tilde{p}_{s} m_{w} \delta_{21} \delta_{22} \dot{\zeta} \frac{W(T)}{\zeta} d \sigma d \zeta\right| \\
& \quad \leq C \int_{\Omega} m_{w}^{2} d \sigma d \zeta+\varepsilon \int_{\Omega}\left|\nabla \cdot\left(\tilde{p}_{s} \bar{V}\right)\right|^{2} d \sigma d \zeta+\varepsilon \int_{\Omega}\left(\frac{1}{\zeta} \int_{0}^{\zeta} \nabla \cdot\left(\tilde{p}_{s} V\right) d s\right)^{2} d \sigma d \zeta \\
& \quad \leq C \int_{\Omega} m_{w}^{2} d \sigma d \zeta+C \int_{\Omega}|V|^{2} d \sigma d \zeta+\varepsilon C \int_{\Omega}|\nabla V|^{2} d \sigma d \zeta \\
& \left|\int_{\Omega} \tilde{p}_{s} m_{w} h_{1}\left(\delta_{21} \delta_{22} \dot{\zeta} \frac{W(T)}{\zeta}\right) d \sigma d \zeta\right| \\
& \quad \leq C \int_{\Omega} m_{w}^{2} d \sigma d \zeta+\varepsilon \int_{\Omega}\left|\nabla \cdot\left(\tilde{p}_{s} \bar{V}\right)\right|^{2} d \sigma d \zeta+\varepsilon \int_{\Omega}\left(\frac{1}{\zeta} \int_{0}^{\zeta} \nabla \cdot\left(\tilde{p}_{s} V\right) d s\right)^{2} d \sigma d \zeta \\
& \quad \leq C \int_{\Omega} m_{w}^{2} d \sigma d \zeta+C \int_{\Omega}|V|^{2} d \sigma d \zeta+\varepsilon C \int_{\Omega}|\nabla V|^{2} d \sigma d \zeta \\
& \left|\int_{S^{2}} \tilde{p}_{s}\left(f\left(\left|V_{10}\right|\right)\left(\frac{g \zeta}{R \widetilde{T}}\right)^{2} q_{m}^{*} q\right)\right|_{\zeta=1} d \sigma\left|\leq C+\varepsilon \int_{S^{2}} f\left(\left|V_{10}\right|\right) q^{2}\right|_{\zeta=1} d \sigma
\end{aligned}
$$

where $C>0$ denotes a constant independent of time $M$.

Using (4.18)-(4.22) and the Young inequality, we deduce

$$
\begin{aligned}
& \frac{d}{d t} \int_{\Omega} \widetilde{p}_{s}\left(V^{2}+\frac{R}{c_{0}^{2}} T^{2}+q^{2}+m_{w}^{2}\right) d \sigma d \zeta \\
& \quad+\frac{d}{d t} \int_{S^{2}} \frac{R \widetilde{T}_{s}}{\widetilde{p}_{s}}\left(\int_{0}^{t} \nabla \cdot\left(\widetilde{p}_{s} \bar{V}\right) d \tau\right)^{2} d \sigma+C\|U\|_{H^{1}(\Omega)}^{2} \\
& \quad+C\left\|T^{\prime}\right\|_{L^{2}(\Omega)}^{2}+\left.C \int_{S^{2}} f(|V|)|V|^{2}\right|_{\zeta=1} d \sigma \\
& \quad+\left.C \int_{S^{2}} T^{\prime 2}\right|_{\zeta=1} d \sigma+\left.C \int_{S^{2}} f\left(\left|V_{10}\right|\right) q^{2}\right|_{\zeta=1} d \sigma \\
& \leq C+\int_{\Omega} \widetilde{p}_{s}\left(V^{2}+\frac{R}{c_{0}^{2}} T^{\prime 2}+q^{2}+m_{w}^{2}\right) d \sigma d \zeta
\end{aligned}
$$

by applying Gronwall inequality, we can prove (4.16).

Remark 4.2 Note that, from [17], for the strong solution $V, T^{\prime}$ to the system $(2.4)_{1,2}$,

$$
\begin{aligned}
& \int_{\Omega}|\tilde{V}|^{4} d \sigma d \zeta+\int_{0}^{t} \int_{\Omega}|\nabla \tilde{V}|^{2}|\tilde{V}|^{2} d \sigma d \zeta d \tau+\int_{0}^{t} \int_{\Omega}\left|\frac{\partial \tilde{V}}{\partial \zeta}\right|^{2}|\tilde{V}|^{2} d \sigma d \zeta d \tau \\
& +\left.\int_{0}^{t} \int_{S^{2}}|\widetilde{V}|^{4+\alpha}\right|_{\zeta=1} d \sigma d \tau \leq C(M), \\
& \int_{S^{2}}|\nabla \bar{V}|^{2} d \sigma+\int_{S^{2}}\left|\int_{0}^{t} \nabla \nabla \cdot\left(\widetilde{p_{s}} \bar{V}\right) d \tau\right|^{2} d \sigma+\int_{0}^{t} \int_{S^{2}}|\Delta \bar{V}|^{2} d \sigma d \tau \leq C(M), \\
& \int_{\Omega}\left|V_{\zeta}\right|^{2} d \sigma d \zeta+\int_{0}^{t} \int_{\Omega}\left|\nabla V_{\zeta}\right|^{2} d \sigma d \zeta d \tau+\int_{0}^{t} \int_{\Omega}\left|V_{\zeta \zeta}\right|^{2} d \sigma d \zeta d \tau
\end{aligned}
$$




$$
\begin{aligned}
& +\left.\int_{S^{2}}\left(f(|V|)|\nabla V|^{2}\right)\right|_{\zeta=1} d \sigma+\left.\int_{S^{2}}\left(\frac{f^{\prime}(|V|)}{|V|}|V \cdot \nabla V|^{2}\right)\right|_{\zeta=1} d \sigma \leq C(M), \\
& \int_{\Omega} T_{\zeta}^{\prime 2} d \sigma d \zeta+\left.\int_{S^{2}} T^{\prime}\right|_{\zeta=1} ^{2} d \sigma+\int_{0}^{t} \int_{\Omega}\left|\nabla T_{\zeta}^{\prime}\right|^{2} d \sigma d \zeta d \tau+\int_{0}^{t} \int_{\Omega} T_{\zeta \zeta}^{\prime 2} d \sigma d \zeta d \tau \\
& \quad+\left.\int_{0}^{t} \int_{S^{2}}\left|\nabla T^{\prime}\right|^{2}\right|_{\zeta=1} d \sigma d \tau \leq C(M), \\
& \int_{\Omega}\left|\nabla\left(\widetilde{p_{s}} V\right)\right|^{2} d \sigma d \zeta+\int_{S^{2}}\left(\int_{0}^{t} \nabla \nabla \cdot\left(\widetilde{p_{s} V}\right) d \tau\right)^{2} d \sigma+\int_{0}^{t} \int_{\Omega}|\Delta V|^{2} d \sigma d \zeta d \tau \\
& \quad+\int_{0}^{t} \int_{\Omega}\left|\nabla V_{\zeta}\right|^{2} d \sigma d \zeta d \tau+\left.\int_{S^{2}}\left(f(|V|)\left|\nabla\left(\widetilde{p_{s}} V\right)\right|^{2}\right)\right|_{\zeta=1} d \sigma \\
& \quad+\left.\int_{S^{2}}\left(\frac{f^{\prime}(|V|)}{|V|}\left|V \cdot \nabla\left(\widetilde{p_{s}} V\right)\right|^{2}\right)\right|_{\zeta=1} d \sigma \leq C(M), \\
& \int_{\Omega}\left|\nabla T^{\prime}\right|^{2} d \sigma d \zeta+\int_{0}^{t} \int_{\Omega}\left|\Delta T^{\prime}\right|^{2} d \sigma d \zeta d \tau+\int_{0}^{t} \int_{\Omega}\left|\nabla T_{\zeta}^{\prime}\right|^{2} d \sigma d \zeta d \tau \\
& \quad+\left.\int_{0}^{t} \int_{S^{2}}\left|\nabla T^{\prime}\right|^{2}\right|_{\zeta=1} d \sigma d \tau \leq C(M),
\end{aligned}
$$

and we omit the details of proof here.

Lemma 4.3 Under the assumptions of Theorem 2.1, for any $M>0$ given, the specific humidity $q$ to the system $(2.4)_{3}$ satisfies

$$
\begin{aligned}
& \int_{\Omega}|q|^{3} d \sigma d \zeta+\int_{0}^{t} \int_{\Omega}|\nabla q|^{2}|q| d \sigma d \xi d \tau+\int_{0}^{t} \int_{\Omega}\left|\frac{\partial q}{\partial \zeta}\right|^{2}|q| d \sigma d \xi d \tau \\
& +\left.\int_{0}^{t} \int_{S^{2}} f\left(\left|V_{10}\right|\right)|q|^{3}\right|_{\zeta=1} d \sigma d \tau \leq C(M), \quad t \in[0, M]
\end{aligned}
$$

where $C(M)>0$ denotes a constant dependent of time $M$.

Proof Multiplying $(2.4)_{3}$ by $\tilde{p}_{s}|q| q$ and integrating the result over $\Omega$, we get

$$
\begin{aligned}
& \frac{1}{3} \frac{d}{d t} \int_{\Omega} \widetilde{p}_{s}|q|^{3} d \sigma d \zeta+2 \mu_{3} \int_{\Omega}|\nabla q|^{2}|q| d \sigma d \zeta+2 \nu_{3} \int_{\Omega} \tilde{p}_{s}\left|\frac{\partial q}{\partial \zeta}\right|^{2}|q| d \sigma d \zeta \\
& \quad+\left.k_{s 3} \int_{S^{2}} \tilde{p}_{s}\left(\left(\frac{g \zeta}{R \widetilde{T}}\right)^{2} f\left(\left|V_{10}\right|\right)|q|^{3}\right)\right|_{\zeta=1} d \sigma \\
& =\frac{1}{3} \int_{\Omega} \frac{d \widetilde{p_{s}}}{d t}|q|^{3} d \sigma d \zeta-\int_{\Omega}\left(\left(V^{*} \cdot \nabla\right) q+\dot{\zeta}^{*} \frac{\partial q}{\partial \zeta}\right) \widetilde{p}_{s}|q| q d \sigma d \zeta \\
& \quad+\left.\int_{S^{2}} \widetilde{p}_{s}\left(\left(\frac{g \zeta}{R \widetilde{T}}\right)^{2} f\left(\left|V_{10}\right|\right) q_{m}^{*}|q| q\right)\right|_{\zeta=1} d \sigma \\
& \quad+\int_{\Omega} \widetilde{p}_{s} \delta_{21} \delta_{22} \dot{\zeta} \frac{W(T)}{\zeta}|q| q d \sigma d \zeta .
\end{aligned}
$$

By virtue of (3.5) and (4.16), the Cauchy-Schwarz inequality, the Hardy inequality, the Gagliardo-Nirenberg-Sobolev inequality and the Young inequality, we find that

$$
\int_{\Omega}\left(\left(V^{*} \cdot \nabla\right) q+\dot{\zeta}^{*} \frac{\partial q}{\partial \zeta}\right) \tilde{p}_{s}|q| q d \sigma d \zeta=0
$$




$$
\begin{aligned}
& \left.\left.\left|\int_{S^{2}} \widetilde{p}_{s}\left(\left(\frac{g \zeta}{R \widetilde{T}}\right)^{2} f\left(\left|V_{10}\right|\right) q_{m}^{*}|q| q\right)\right|_{\zeta=1} d \sigma\left|\leq C+\varepsilon \int_{S^{2}} f\left(\left|V_{10}\right|\right)\right| q\right|^{3}\right|_{\zeta=1} d \sigma, \\
& \left|\int_{\Omega} \nabla \cdot\left(\widetilde{p}_{s} \bar{V}\right)(W(T)|q| q) d \sigma d \zeta\right| \\
& \leq C\|V\|_{L^{2}(\Omega)}^{2}+C\|\nabla V\|_{L^{2}(\Omega)}^{2}+C\|q\|_{L^{4}(\Omega)}^{4} \\
& \leq C(M)+C\left\|q^{\frac{3}{2}}\right\|_{L^{\frac{8}{3}}(\Omega)}^{\frac{8}{3}} \\
& \leq C(M)+C\left(\left\|q^{\frac{3}{2}}\right\|_{L^{\frac{4}{3}(\Omega)}}^{\frac{5}{14}}\left(\left\|q^{\frac{3}{2}}\right\|_{L^{2}(\Omega)}+\left\|\nabla\left(q^{\frac{3}{2}}\right)\right\|_{L^{2}(\Omega)}+\left\|\frac{\partial\left(q^{\frac{3}{2}}\right)}{\partial \zeta}\right\|_{L^{2}(\Omega)}\right)^{\frac{9}{14}}\right)^{\frac{8}{3}} \\
& \leq C(M)+C\left(\left\|q^{\frac{3}{2}}\right\|_{L^{2}(\Omega)}+\left\|\nabla\left(q^{\frac{3}{2}}\right)\right\|_{L^{2}(\Omega)}+\left\|\frac{\partial\left(q^{\frac{3}{2}}\right)}{\partial \zeta}\right\|_{L^{2}(\Omega)}\right)^{\frac{12}{7}} \\
& \leq C(M)+C \int_{\Omega} \widetilde{p}_{s}|q|^{3} d \sigma d \zeta+\frac{2 \varepsilon}{9}\left(\left\|\nabla\left(q^{\frac{3}{2}}\right)\right\|_{L^{2}(\Omega)}+\left\|\frac{\partial\left(q^{\frac{3}{2}}\right)}{\partial \zeta}\right\|_{L^{2}(\Omega)}\right)^{2} \\
& \leq C(M)+C \int_{\Omega} \widetilde{p}_{s}|q|^{3} d \sigma d \zeta+\varepsilon \int_{\Omega}|\nabla q|^{2}|q| d \sigma d \zeta+\varepsilon \int_{\Omega}\left|\frac{\partial q}{\partial \zeta}\right|^{2}|q| d \sigma d \zeta, \\
& \left|\int_{\Omega} \delta_{21} \delta_{22}\left(\frac{1}{\zeta} \int_{0}^{\zeta} \nabla \cdot\left(\widetilde{p}_{s} V\right) d s\right)\right| q|q d \sigma d \zeta| \\
& \leq C\left\|\frac{1}{\zeta} \int_{0}^{\zeta} \nabla \cdot\left(\widetilde{p}_{s} V\right) d s\right\|_{L^{2}(\Omega)}^{2}+C\|q\|_{L^{4}(\Omega)}^{4} \\
& \leq C(M)+C \int_{\Omega} \tilde{p}_{s}|q|^{3} d \sigma d \zeta+\varepsilon \int_{\Omega}|\nabla q|^{2}|q| d \sigma d \zeta+\varepsilon \int_{\Omega}\left|\frac{\partial q}{\partial \zeta}\right|^{2}|q| d \sigma d \zeta, \\
& \left|\int_{\Omega} \widetilde{p}_{s} \delta_{21} \delta_{22} \dot{\zeta} \frac{W(T)}{\zeta}\right| q|q d \sigma d \zeta| \\
& \leq C\left|\int_{\Omega} \nabla \cdot\left(\tilde{p}_{s} \bar{V}\right) W(T)\right| q|q d \sigma d \zeta| \\
& +C\left|\int_{\Omega}\left(\frac{1}{\zeta} \int_{0}^{\zeta} \nabla \cdot\left(\widetilde{p_{s}} V\right) d s\right) W(T)\right| q|q d \sigma d \zeta| \\
& \leq C(M)+C \int_{\Omega} \tilde{p}_{s}|q|^{3} d \sigma d \zeta+\varepsilon \int_{\Omega}|\nabla q|^{2}|q| d \sigma d \zeta+\varepsilon \int_{\Omega}\left|\frac{\partial q}{\partial \zeta}\right|^{2}|q| d \sigma d \zeta,
\end{aligned}
$$

where $C(M)>0$ denotes a constant dependent of time $M$ and $\varepsilon>0$ is a small constant such that

$$
\begin{aligned}
& \frac{d}{d t} \int_{\Omega} \tilde{p}_{s}|q|^{3} d \sigma d \zeta+C \int_{\Omega}|\nabla q|^{2}|q| d \sigma d \zeta+C \int_{\Omega}\left|\frac{\partial q}{\partial \zeta}\right|^{2}|q| d \sigma d \zeta \\
& \quad+\left.C \int_{S^{2}} f\left(\left|V_{10}\right|\right)|q|^{3}\right|_{\zeta=1} d \sigma \\
& \leq C(M)+C \int_{\Omega} \widetilde{p}_{s}|q|^{3} d \sigma d \zeta,
\end{aligned}
$$

by applying the Gronwall inequality, we get (4.30). 
Lemma 4.4 Under the assumptions of Theorem 2.1, for any $M>0$ given, the liquid water content $m_{w}$ to the system $(2.4)_{4}$ satisfies

$$
\begin{aligned}
& \int_{\Omega}\left|m_{w}\right|^{3} d \sigma d \zeta+\int_{0}^{t} \int_{\Omega}\left|\nabla m_{w}\right|^{2}\left|m_{w}\right| d \sigma d \xi d \tau+\int_{0}^{t} \int_{\Omega}\left|\frac{\partial m_{w}}{\partial \zeta}\right|^{2}\left|m_{w}\right| d \sigma d \xi d \tau \\
& \quad \leq C(M), \quad t \in[0, M]
\end{aligned}
$$

where $C(M)>0$ denotes a constant dependent of time $M$.

Proof Multiplying (2.4) $)_{4}$ by $\widetilde{p}_{s}\left|m_{w}\right| m_{w}$ and integrating the result over $\Omega$, we have

$$
\begin{aligned}
& \frac{1}{3} \frac{d}{d t} \int_{\Omega} \widetilde{p}_{s}\left|m_{w}\right|^{3} d \sigma d \zeta+2 \mu_{3} \int_{\Omega}\left|\nabla m_{w}\right|^{2}\left|m_{w}\right| d \sigma d \zeta+2 v_{4} \int_{\Omega} \widetilde{p}_{s}\left|\frac{\partial m_{w}}{\partial \zeta}\right|^{2}\left|m_{w}\right| d \sigma d \zeta \\
& =\frac{1}{3} \int_{\Omega} \frac{d \widetilde{p}_{s}}{d t}\left|m_{w}\right|^{3} d \sigma d \zeta-\int_{\Omega}\left(\left(V^{*} \cdot \nabla\right) m_{w}+\dot{\zeta} \frac{\partial m_{w}}{\partial \zeta}\right) \widetilde{p}_{s}\left|m_{w}\right| m_{w} d \sigma d \zeta \\
& \quad+\int_{\Omega} \widetilde{p}_{s} \delta_{21} \delta_{22} \dot{\zeta} \frac{W(T)}{\zeta}\left|m_{w}\right| m_{w} d \sigma d \zeta \\
& \quad+\int_{\Omega} \widetilde{p}_{s} h_{1}\left(\delta_{21} \delta_{22} \dot{\zeta} \frac{W(T)}{\zeta}\right)\left|m_{w}\right| m_{w} d \sigma d \zeta
\end{aligned}
$$

Thanks to (3.6), the Cauchy-Schwarz inequality, the Hardy inequality, the GagliardoNirenberg-Sobolev inequality and the Young inequality, we know that

$$
\begin{aligned}
& \int_{\Omega}\left(\left(V^{*} \cdot \nabla\right) m_{w}+\dot{\zeta} * \frac{\partial m_{w}}{\partial \zeta}\right) \tilde{p}_{s}\left|m_{w}\right| m_{w} d \sigma d \zeta=0 \\
& \left|\int_{\Omega} \nabla \cdot\left(\tilde{p}_{s} \bar{V}\right) W(T)\right| m_{w}\left|m_{w} d \sigma d \zeta\right| \\
& \quad \leq C\left(\|V\|_{L^{2}(\Omega)}^{2}+\|\nabla V\|_{L^{2}(\Omega)}^{2}\right)+C\left\|m_{w}\right\|_{L^{4}(\Omega)}^{4} \\
& \quad \leq C(M)+C\left\|m_{w}^{\frac{3}{2}}\right\|_{L^{\frac{8}{3}}(\Omega)}^{\frac{8}{3}} \\
& \quad \leq C(M)+C\left(\left\|m_{w}^{\frac{3}{2}}\right\|_{L^{\frac{4}{3}}(\Omega)}^{\frac{5}{14}}\left(\left\|m_{w}^{\frac{3}{2}}\right\|_{L^{2}(\Omega)}+\left\|\nabla\left(m_{w}^{\frac{3}{2}}\right)\right\|_{L^{2}(\Omega)}+\left\|\frac{\partial\left(m_{w}^{\frac{3}{2}}\right)}{\partial \zeta}\right\|_{L^{2}(\Omega)}\right)^{\frac{9}{14}}\right)^{\frac{8}{3}} \\
& \quad \leq C(M)+C\left(\left\|m_{w}^{\frac{3}{2}}\right\|_{L^{2}(\Omega)}+\left\|\nabla\left(m_{w}^{\frac{3}{2}}\right)\right\|_{L^{2}(\Omega)}+\left\|\frac{\partial\left(m_{w}^{\frac{3}{2}}\right)}{\partial \zeta}\right\|_{L^{2}(\Omega)}\right)^{\frac{12}{7}} \\
& \quad \leq C(M)+C \int_{\Omega} \widetilde{p}_{s}\left|m_{w}\right|^{3} d \sigma d \zeta+\frac{2 \varepsilon}{9}\left(\left\|\nabla\left(m_{w}^{\frac{3}{2}}\right)\right\|_{L^{2}(\Omega)}+\left\|\frac{\partial\left(m_{w}^{\frac{3}{2}}\right)}{\partial \zeta}\right\|_{L^{2}(\Omega)}\right)^{2} \\
& \quad \leq C(M)+C \int_{\Omega} \widetilde{p}_{s}\left|m_{w}\right|^{3} d \sigma d \zeta+\varepsilon \int_{\Omega}\left|\nabla m_{w}\right|^{2}\left|m_{w}\right| d \sigma d \zeta \\
& \quad+\varepsilon \int_{\Omega}\left|\frac{\partial m_{w}}{\partial \zeta}\right|^{2}\left|m_{w}\right| d \sigma d \zeta \\
& \left|\int_{\Omega} \delta_{21} \delta_{22}\left(\frac{1}{\zeta} \int_{0}^{\zeta} \nabla \cdot\left(\widetilde{p}_{s} V\right) d s\right)\right| m_{w}\left|m_{w} d \sigma d \zeta\right|
\end{aligned}
$$




$$
\begin{aligned}
& \leq C\left\|\frac{1}{\zeta} \int_{0}^{\zeta} \nabla \cdot\left(\widetilde{p}_{s} V\right) d s\right\|_{L^{2}(\Omega)}^{2}+C\left\|m_{w}\right\|_{L^{4}(\Omega)}^{4} \\
& \leq C(M)+C \int_{\Omega} \widetilde{p}_{s}\left|m_{w}\right|^{3} d \sigma d \zeta+\varepsilon \int_{\Omega}\left|\nabla m_{w}\right|^{2}\left|m_{w}\right| d \sigma d \zeta \\
&+\varepsilon \int_{\Omega}\left|\frac{\partial m_{w}}{\partial \zeta}\right|^{2}\left|m_{w}\right| d \sigma d \zeta, \\
&\left|\int_{\Omega} \widetilde{p}_{s} \delta_{21} \delta_{22} \dot{\zeta} \frac{W(T)}{\zeta}\right| m_{w}\left|m_{w} d \sigma d \zeta\right| \\
& \leq C\left|\int_{\Omega} \nabla \cdot\left(\widetilde{p_{s}} \bar{V}\right)\left(W(T)\left|m_{w}\right| m_{w}\right) d \sigma d \zeta\right| \\
& \quad+C\left|\int_{\Omega}\left(\frac{1}{\zeta} \int_{0}^{\zeta} \nabla \cdot\left(\widetilde{p}_{s} V\right) d s\right) W(T)\right| m_{w}\left|m_{w} d \sigma d \zeta\right| \\
& \leq C(M)+C \int_{\Omega} \widetilde{p}_{s}\left|m_{w}\right|^{3} d \sigma d \zeta+\varepsilon \int_{\Omega}\left|\nabla m_{w}\right|^{2}\left|m_{w}\right| d \sigma d \zeta \\
&+\varepsilon \int_{\Omega}\left|\frac{\partial m_{w}}{\partial \zeta}\right|^{2}\left|m_{w}\right| d \sigma d \zeta,
\end{aligned}
$$

where $C(M)>0$ denotes a constant dependent of time $M$ and $\varepsilon>0$ is a small constant such that

$$
\begin{aligned}
& \frac{d}{d t} \int_{\Omega} \widetilde{p}_{s}\left|m_{w}\right|^{3} d \sigma d \zeta+C \int_{\Omega}\left|\nabla m_{w}\right|^{2}\left|m_{w}\right| d \sigma d \zeta+C \int_{\Omega}\left|\frac{\partial m_{w}}{\partial \zeta}\right|^{2}\left|m_{w}\right| d \sigma d \zeta \\
& \quad \leq C(M)+C \int_{\Omega} \widetilde{p}_{s}\left|m_{w}\right|^{3} d \sigma d \zeta
\end{aligned}
$$

using the Gronwall inequality, we can obtain (4.38).

Lemma 4.5 Under the assumptions of Theorem 2.1, for any $M>0$ given, the specific humidity $q$ to the system $(2.4)_{3}$ satisfies

$$
\begin{gathered}
\int_{\Omega} q^{4} d \sigma d \zeta+\int_{0}^{t} \int_{\Omega}|\nabla q|^{2} q^{2} d \sigma d \xi d \tau+\int_{0}^{t} \int_{\Omega}\left|\frac{\partial q}{\partial \zeta}\right|^{2} q^{2} d \sigma d \xi d \tau \\
+\left.\int_{0}^{t} \int_{S^{2}} f\left(\left|V_{10}\right|\right) q^{4}\right|_{\zeta=1} d \sigma d \tau \leq C(M), \quad t \in[0, M]
\end{gathered}
$$

where $C(M)>0$ denotes a constant dependent of time $M$.

Proof Taking the inner product of the $(2.4)_{3}$ with $\widetilde{p_{s}} q^{3}$ and integrating the result over $\Omega$, we get

$$
\begin{aligned}
& \frac{1}{4} \frac{d}{d t} \int_{\Omega} \widetilde{p}_{s} q^{4} d \sigma d \zeta+3 \mu_{3} \int_{\Omega}|\nabla q|^{2} q^{2} d \sigma d \zeta+3 v_{3} \int_{\Omega} \widetilde{p}_{s}\left|\frac{\partial q}{\partial \zeta}\right|^{2} q^{2} d \sigma d \zeta \\
& \quad+\left.\int_{S^{2}} \widetilde{p_{s}}\left(f\left(\left|V_{10}\right|\right)\left(\frac{g \zeta}{R \widetilde{T}}\right)^{2} q^{4}\right)\right|_{\zeta=1} d \sigma \\
& =\frac{1}{4} \int_{\Omega} \frac{d \widetilde{p_{s}}}{d t} q^{4} d \sigma d \zeta-\int_{\Omega}\left(\left(V^{*} \cdot \nabla\right) q+\zeta^{*} \frac{\partial q}{\partial \zeta}\right) \widetilde{p}_{s} q^{3} d \sigma d \zeta
\end{aligned}
$$




$$
+\left.\int_{S^{2}} \widetilde{p}_{s}\left(\left(\frac{g \zeta}{R \widetilde{T}}\right)^{2} f\left(\left|V_{10}\right|\right) q_{m}^{*} q^{3}\right)\right|_{\zeta=1} d \sigma+\int_{\Omega} \widetilde{p}_{s} \delta_{21} \delta_{22} \dot{\zeta} \frac{W(T)}{\zeta} q^{3} d \sigma d \zeta
$$

Using (3.5), the Cauchy-Schwarz inequality, the Hardy inequality, the GagliardoNirenberg-Sobolev inequality and the Young inequality, we know that

$$
\begin{aligned}
& \int_{\Omega}\left(\left(V^{*} \cdot \nabla\right) q+\dot{\zeta} * \frac{\partial q}{\partial \zeta}\right) \tilde{p}_{s} q^{3} d \sigma d \zeta=0, \\
& \left|\int_{S^{2}} \widetilde{p}_{s}\left(\frac{g \zeta}{R \widetilde{T}}\right)^{2} f\left(\left|V_{10}\right|\right) q_{m}^{*} q^{3}\right|_{\zeta=1} d \sigma\left|\leq C+\varepsilon \int_{S^{2}} f\left(\left|V_{10}\right|\right) q^{4}\right|_{\zeta=1} d \sigma, \\
& \left|\int_{\Omega} \nabla \cdot\left(\widetilde{p}_{s} \bar{V}\right) W(T) q^{3} d \sigma d \zeta\right| \\
& \leq C\left(\|V\|_{L^{2}(\Omega)}+\|\nabla V\|_{L^{2}(\Omega)}\right)\|q\|_{L^{6}(\Omega)}^{3} \\
& \leq C(M)\left(\left\|q^{2}\right\|_{L^{\frac{3}{2}(\Omega)}}^{\frac{1}{3}}\left(\left\|q^{2}\right\|_{L^{2}(\Omega)}+\left\|\nabla\left(q^{2}\right)\right\|_{L^{2}(\Omega)}+\left\|\frac{\partial q^{2}}{\partial \zeta}\right\|_{L^{2}(\Omega)}\right)^{\frac{2}{3}}\right)^{\frac{3}{2}} \\
& \leq C(M)\left(\left\|q^{2}\right\|_{L^{2}(\Omega)}+\left\|\nabla\left(q^{2}\right)\right\|_{L^{2}(\Omega)}+\left\|\frac{\partial q^{2}}{\partial \zeta}\right\|_{L^{2}(\Omega)}\right) \\
& \leq C(M)+C \int_{\Omega} \tilde{p}_{s} q^{4} d \sigma d \zeta+\varepsilon \int_{\Omega}|\nabla q|^{2} q^{2} d \sigma d \zeta+\varepsilon \int_{\Omega}\left|\frac{\partial q}{\partial \zeta}\right|^{2} q^{2} d \sigma d \zeta, \\
& \left|\int_{\Omega}\left(\frac{1}{\zeta} \int_{0}^{\zeta} \nabla \cdot\left(\tilde{p}_{s} V\right) d s\right) W(T) q^{3} d \sigma d \zeta\right| \\
& \leq C\left(\int_{\Omega}\left(\frac{1}{\zeta} \int_{0}^{\zeta} \nabla \cdot\left(\widetilde{p}_{s} V\right) d s\right)^{2} d \sigma d \zeta\right)^{\frac{1}{2}}\left(\int_{\Omega} q^{6} d \sigma d \zeta\right)^{\frac{1}{2}} \\
& \leq C(M)\left(\int_{\Omega}\left|\nabla \cdot\left(\widetilde{p}_{s} V\right)\right|^{2} d \sigma d \zeta\right)^{\frac{1}{2}}\left(\left\|q^{2}\right\|_{L^{2}(\Omega)}+\left\|\nabla\left(q^{2}\right)\right\|_{L^{2}(\Omega)}+\left\|\frac{\partial q^{2}}{\partial \zeta}\right\|_{L^{2}(\Omega)}\right) \\
& \leq C(M)+C \int_{\Omega} \tilde{p}_{s} q^{4} d \sigma d \zeta+\varepsilon \int_{\Omega}|\nabla q|^{2} q^{2} d \sigma d \zeta \\
& +\varepsilon \int_{\Omega}\left|\frac{\partial q}{\partial \zeta}\right|^{2} q^{2} d \sigma d \zeta \\
& \left|\int_{\Omega} \tilde{p}_{s} \delta_{21} \delta_{22} \dot{\zeta} \frac{W(T)}{\zeta} q^{3} d \sigma d \zeta\right| \\
& \leq C(M)+C \int_{\Omega} \tilde{p}_{s} q^{4} d \sigma d \zeta+\varepsilon \int_{\Omega}|\nabla q|^{2} q^{2} d \sigma d \zeta \\
& +\varepsilon \int_{\Omega}\left|\frac{\partial q}{\partial \zeta}\right|^{2} q^{2} d \sigma d \zeta
\end{aligned}
$$

where $C(M)>0$ denotes a constant dependent of time $M$ and $\varepsilon>0$ is a small constant such that

$$
\begin{aligned}
& \frac{d}{d t} \int_{\Omega} \widetilde{p}_{s} q^{4} d \sigma d \zeta+C \int_{\Omega}|\nabla q|^{2} q^{2} d \sigma d \zeta+C \int_{\Omega}\left|\frac{\partial q}{\partial \zeta}\right|^{2} q^{2} d \sigma d \zeta \\
& \quad+\left.C \int_{S^{2}} f\left(\left|V_{10}\right|\right) q^{4}\right|_{\zeta=1} d \sigma
\end{aligned}
$$




$$
\leq C(M)+C \int_{\Omega} \tilde{p}_{s} q^{4} d \sigma d \zeta
$$

applying the Gronwall inequality, we deduce (4.45).

Lemma 4.6 Under the assumptions of Theorem 2.1, for any $M>0$ given, the liquid water content $m_{w}$ to the system $(2.4)_{4}$ satisfies

$$
\begin{aligned}
& \int_{\Omega} m_{w}^{4} d \sigma d \zeta+\int_{0}^{t} \int_{\Omega}\left|\nabla m_{w}\right|^{2} m_{w}^{2} d \sigma d \xi d \tau+\int_{0}^{t} \int_{\Omega}\left|\frac{\partial m_{w}}{\partial \zeta}\right|^{2} m_{w}^{2} d \sigma d \xi d \tau \\
& \quad \leq C(M), \quad t \in[0, M]
\end{aligned}
$$

where $C(M)>0$ denotes a constant dependent of time $M$.

Proof Multiplying $(2.4)_{4}$ by $\widetilde{p}_{s} m_{w}^{3}$ and integrating the result over $\Omega$, we know

$$
\begin{aligned}
& \frac{1}{4} \frac{d}{d t} \int_{\Omega} \widetilde{p}_{s} m_{w}^{4} d \sigma d \zeta+3 \mu_{4} \int_{\Omega}\left|\nabla m_{w}\right|^{2} m_{w}^{2} d \sigma d \zeta \\
& \quad+3 v_{4} \int_{\Omega} \widetilde{p}_{s}\left|\frac{\partial m_{w}}{\partial \zeta}\right|^{2} m_{w}^{2} d \sigma d \zeta+\left.\int_{S^{2}} \widetilde{p}_{s}\left(\left(\frac{g \zeta}{R \widetilde{T}}\right)^{2} m_{w}^{4}\right)\right|_{\zeta=1} d \sigma \\
& =\frac{1}{4} \int_{\Omega} \frac{d \widetilde{p_{s}}}{d t} m_{w}^{4} d \sigma d \zeta-\int_{\Omega}\left(\left(V^{*} \cdot \nabla\right) m_{w}+\dot{\zeta}^{*} \frac{\partial m_{w}}{\partial \zeta}\right) \widetilde{p}_{s} m_{w}^{3} d \sigma d \zeta \\
& \quad+\int_{\Omega} \widetilde{p}_{s} \delta_{21} \delta_{22} \dot{\zeta} \frac{W(T)}{\zeta} m_{w}^{3} d \sigma d \zeta+\int_{\Omega} \widetilde{p}_{s} h_{1}\left(\delta_{21} \delta_{22} \dot{\zeta} \frac{W(T)}{\zeta}\right) m_{w}^{3} d \sigma d \zeta
\end{aligned}
$$

Thanks to (3.6), the Cauchy-Schwarz inequality, the Hardy inequality, the GagliardoNirenberg-Sobolev inequality and the Young inequality, we get

$$
\begin{aligned}
& \int_{\Omega}\left(\left(V^{*} \cdot \nabla\right) m_{w}+\dot{\zeta} * \frac{\partial m_{w}}{\partial \zeta}\right) \tilde{p}_{s} m_{w}^{3} d \sigma d \zeta=0 \\
& \left|\int_{\Omega} \nabla \cdot\left(\widetilde{p_{s}} \bar{V}\right) W(T) m_{w}^{3} d \sigma d \zeta\right| \\
& \quad \leq C\left(\|V\|_{L^{2}(\Omega)}+\|\nabla V\|_{L^{2}(\Omega)}\right)\left\|m_{w}\right\|_{L^{6}(\Omega)}^{3} \\
& \quad \leq C(M)\left(\left\|m_{w}^{2}\right\|_{L^{2} \frac{1}{3}(\Omega)}^{\frac{1}{3}}\left(\left\|m_{w}^{2}\right\|_{L^{2}(\Omega)}+\left\|\nabla\left(m_{w}^{2}\right)\right\|_{L^{2}(\Omega)}+\left\|\frac{\partial m_{w}^{2}}{\partial \zeta}\right\|_{L^{2}(\Omega)}\right)^{\frac{2}{3}}\right)^{\frac{3}{2}} \\
& \quad \leq C(M)\left(\left\|m_{w}^{2}\right\|_{L^{2}(\Omega)}+\left\|\nabla\left(m_{w}^{2}\right)\right\|_{L^{2}(\Omega)}+\left\|\frac{\partial m_{w}^{2}}{\partial \zeta}\right\|_{L^{2}(\Omega)}\right) \\
& \quad \leq C(M)+C \int_{\Omega} \tilde{p}_{s} m_{w}^{4} d \sigma d \zeta+\varepsilon \int_{\Omega}\left|\nabla m_{w}\right|^{2} m_{w}^{2} d \sigma d \zeta \\
& \quad+\varepsilon \int_{\Omega}\left|\frac{\partial m_{w}}{\partial \zeta}\right|^{2} m_{w}^{2} d \sigma d \zeta, \\
& \quad \leq C\left\|\frac{1}{\zeta} \int_{0}^{\zeta} \nabla \cdot\left(\tilde{p}_{s} V\right) d s\right\| \\
& \quad\left(\int_{L^{2}(\Omega)}^{\zeta} \nabla \cdot\left(\tilde{p}_{s} V\right) d s\right) W(T) m_{w}^{3} d \sigma d \zeta \mid
\end{aligned}
$$




$$
\begin{aligned}
& \leq C(M)\left(\left\|m_{w}^{2}\right\|_{L^{2}(\Omega)}+\left\|\nabla m_{w}^{2}\right\|_{L^{2}(\Omega)}+\left\|\frac{\partial m_{w}^{2}}{\partial \zeta}\right\|_{L^{2}(\Omega)}\right) \\
& \leq C(M)+C \int_{\Omega} \widetilde{p}_{s} m_{w}^{4} d \sigma d \zeta+\varepsilon \int_{\Omega}\left|\nabla m_{w}\right|^{2} m_{w}^{2} d \sigma d \zeta \\
&+\varepsilon \int_{\Omega}\left|\frac{\partial m_{w}}{\partial \zeta}\right|^{2} m_{w}^{2} d \sigma d \zeta, \\
&\left|\int_{\Omega} \tilde{p}_{s} \delta_{21} \delta_{22} \dot{\zeta} \frac{W(T)}{\zeta} m_{w}^{3} d \sigma d \zeta\right| \\
& \leq C(M)+C \int_{\Omega} \widetilde{p}_{s} m_{w}^{4} d \sigma d \zeta+\varepsilon \int_{\Omega}\left|\nabla m_{w}\right|^{2} m_{w}^{2} d \sigma d \zeta \\
& \quad+\varepsilon \int_{\Omega}\left|\frac{\partial m_{w}}{\partial \zeta}\right|^{2} m_{w}^{2} d \sigma d \zeta,
\end{aligned}
$$

where $C(M)>0$ denotes a constant dependent of time $M$ and $\varepsilon>0$ is a small constant such that

$$
\begin{aligned}
& \frac{d}{d t} \int_{\Omega} \tilde{p}_{s} m_{w}^{4} d \sigma d \zeta+C \int_{\Omega}\left|\nabla m_{w}\right|^{2} m_{w}^{2} d \sigma d \zeta+C \int_{\Omega}\left|\frac{\partial m_{w}}{\partial \zeta}\right|^{2} m_{w}^{2} d \sigma d \zeta \\
& \quad \leq C(M)+C \int_{\Omega} \tilde{p}_{s} m_{w}^{4} d \sigma d \zeta
\end{aligned}
$$

by applying the Gronwall inequality, we infer (4.53).

Lemma 4.7 Under the assumptions of Theorem 2.1, for any $M>0$ given, the specific humidity $q$ to the system $(2.4)_{3}$ satisfies

$$
\begin{aligned}
& \int_{\Omega} q_{\zeta}^{2} d \sigma d \zeta+\int_{0}^{t} \int_{\Omega}\left|\nabla q_{\zeta}\right|^{2} d \sigma d \zeta d \tau+\int_{0}^{t} \int_{\Omega} q_{\zeta \zeta}^{2} d \sigma d \zeta d \tau+\left.\int_{S^{2}} q^{2}\right|_{\zeta=1} d \sigma \\
& \quad+\left.\int_{0}^{t} \int_{S^{2}}|\nabla q|^{2}\right|_{\zeta=1} d \sigma d \tau \leq C(M), \quad t \in[0, M]
\end{aligned}
$$

where $C(M)>0$ denotes a constant dependent of time $M$.

Proof Taking the derivative with respect to $\zeta$ of $(2.4)_{3}$, we find that

$$
\begin{aligned}
\frac{\partial q_{\zeta}}{\partial t} & -\mu_{3} \frac{1}{\widetilde{p}_{s}} \Delta q_{\zeta}-v_{3} \frac{\partial}{\partial \zeta}\left(\left(\frac{g \zeta}{R \widetilde{T}}\right)^{2} q_{\zeta \zeta}\right) \\
& +\left(\left(V^{*} \cdot \nabla\right) q_{\zeta}+\dot{\zeta}^{*} q_{\zeta \zeta}\right)+\left(\left(V_{\zeta}^{*} \cdot \nabla\right) q-\frac{1}{\widetilde{p}_{s}} \nabla \cdot\left(\widetilde{p_{s}} V^{*}\right) q_{\zeta}\right) \\
= & v_{3} \frac{\partial}{\partial \zeta}\left(\frac{\partial}{\partial \zeta}\left(\left(\frac{g \zeta}{R \widetilde{T}}\right)^{2}\right) q_{\zeta}\right)+\frac{\partial}{\partial \zeta}\left(\delta_{21} \delta_{22} \dot{\zeta} \frac{W(T)}{\zeta}\right) .
\end{aligned}
$$

Multiplying (4.61) by $\widetilde{p_{s}} q_{\zeta}$, we have

$$
\begin{aligned}
\frac{1}{2} & \frac{d}{d t} \int_{\Omega} \widetilde{p}_{s} q_{\zeta}^{2} d \sigma d \zeta+\mu_{3} \int_{\Omega}\left|\nabla q_{\zeta}\right|^{2} d \sigma d \zeta+v_{3} \int_{\Omega} \widetilde{p_{s}}\left(\frac{g \zeta}{R \widetilde{T}}\right)^{2} q_{\zeta \zeta}^{2} d \sigma d \zeta \\
& =\int_{\Omega} \frac{d \widetilde{p_{s}}}{d t} q_{\zeta}^{2} d \sigma d \zeta-\int_{\Omega}\left(\left(V^{*} \cdot \nabla\right) q_{\zeta}+\dot{\zeta}^{*} q_{\zeta \zeta}\right) \tilde{p}_{s} q_{\zeta} d \sigma d \zeta
\end{aligned}
$$




$$
\begin{aligned}
& -\int_{\Omega}\left(\left(V_{\zeta}^{*} \cdot \nabla\right) q-\frac{1}{\widetilde{p}_{s}} \nabla \cdot\left(\widetilde{p}_{s} V^{*}\right) q_{\zeta}-\frac{1}{\widetilde{p_{s}}} \frac{\partial \widetilde{p}_{s}}{\partial t} \zeta q_{\zeta}\right) \tilde{p}_{s} q_{\zeta} d \sigma d \zeta \\
& +v_{3} \int_{\Omega} \frac{\partial}{\partial \zeta}\left(\frac{\partial}{\partial \zeta}\left(\left(\frac{g \zeta}{R \widetilde{T}}\right)^{2}\right) q_{\zeta}\right) \widetilde{p}_{s} q_{\zeta} d \sigma d \zeta \\
& +\left.\left.v_{3} \int_{S^{2}} \widetilde{p}_{s} q_{\zeta}\right|_{\zeta=1}\left(\left(\frac{g \zeta}{R \widetilde{T}}\right)^{2} q_{\zeta \zeta}\right)\right|_{\zeta=1} d \sigma \\
& +\int_{\Omega} \frac{\partial}{\partial \zeta}\left(\delta_{21} \delta_{22} \dot{\zeta} \frac{W(T)}{\zeta}\right) \tilde{p}_{s} q_{\zeta} d \sigma d \zeta .
\end{aligned}
$$

Similarly to (4.32), we know

$$
-\int_{\Omega}\left(\left(V^{*} \cdot \nabla\right) q_{\zeta}+\dot{\zeta}^{*} q_{\zeta \zeta}\right) \tilde{p}_{s} q_{\zeta} d \sigma d \zeta=0
$$

By (4.16), (4.24)-(4.26), (4.45), the Gagliardo-Nirenberg-Sobolev inequality, the Young inequality and the fact that $V_{\zeta}^{*}=V_{\zeta}$, we know that

$$
\begin{aligned}
\mid-\int_{\Omega} & \left(\left(V_{\zeta}^{*} \cdot \nabla\right) q-\frac{1}{\widetilde{p}_{s}} \nabla \cdot\left(\widetilde{p}_{s} V^{*}\right) q_{\zeta}-\frac{1}{\widetilde{p_{s}}} \frac{\partial \widetilde{p}_{s}}{\partial t} \zeta q_{\zeta}\right) \widetilde{p}_{s} q_{\zeta} d \sigma d \zeta \mid \\
\leq & C \int_{\Omega}\left(\left(\left|V_{\zeta}\right|+\left|\nabla V_{\zeta}\right|\right)\left|q\left\|q_{\zeta}|+| V_{\zeta}\right\| q \| \nabla q_{\zeta}\right|+|V|\left|T_{\zeta}^{\prime}\right|\left|\nabla q_{\zeta}\right|\right) d \sigma d \zeta \\
& +C(M)\left\|q_{\zeta}\right\|_{L^{2}(\Omega)}^{2} \\
\leq & C\left\|V_{\zeta}\right\|_{L^{2}(\Omega)}^{2}+C\left\|\nabla V_{\zeta}\right\|_{L^{2}(\Omega)}^{2}+C\|q\|_{L^{4}(\Omega)}^{2}\left\|q_{\zeta}\right\|_{L^{4}(\Omega)}^{2} \\
& +C\left\|V_{\zeta}\right\|_{L^{4}(\Omega)}^{2}\|q\|_{L^{4}(\Omega)}^{2}+C(M)\left\|q_{\zeta}\right\|_{L^{2}(\Omega)}^{2} \\
& +C\|V\|_{L^{4}(\Omega)}^{2}\left\|q_{\zeta}\right\|_{L^{4}(\Omega)}^{2}+\varepsilon\left\|\nabla T_{\zeta}^{\prime}\right\|_{L^{2}(\Omega)}^{2} \\
\leq & C\left\|V_{\zeta}\right\|_{L^{2}(\Omega)}^{2}+C\left\|\nabla V_{\zeta}\right\|_{L^{2}(\Omega)}^{2} \\
& +C\|q\|_{L^{4}(\Omega)}^{2}\left\|q_{\zeta}\right\|_{L^{2}(\Omega)}^{\frac{1}{2}}\left(\left\|q_{\zeta}\right\|_{L^{2}(\Omega)}+\left\|\nabla q_{\zeta}\right\|_{L^{2}(\Omega)}+\left\|q_{\zeta \zeta}\right\|_{L^{2}(\Omega)}\right)^{\frac{3}{2}} \\
& +C\left\|V_{\zeta}\right\|_{L^{2}(\Omega)}^{\frac{1}{2}}\left(\left\|V_{\zeta}\right\|_{L^{2}(\Omega)}+\left\|\nabla V_{\zeta}\right\|_{L^{2}(\Omega)}+\left\|V_{\zeta \zeta}\right\|_{L^{2}(\Omega)}\right)^{\frac{3}{2}}\|q\|_{L^{4}(\Omega)}^{2} \\
& +C\|V\|_{L^{4}(\Omega)}^{2}\left\|q_{\zeta}\right\|_{L^{2}(\Omega)}^{\frac{1}{2}}\left(\left\|q_{\zeta}\right\|_{L^{2}(\Omega)}+\left\|\nabla q_{\zeta}\right\|_{L^{2}(\Omega)}+\left\|q_{\zeta \zeta}\right\|_{L^{2}(\Omega)}\right)^{\frac{3}{2}}+\varepsilon\left\|\nabla q_{\zeta}\right\|_{L^{2}(\Omega)}^{2} \\
& +C(M)\left\|q_{\zeta}\right\|_{L^{2}(\Omega)}^{2} \\
\leq & C\left\|V_{\zeta}\right\|_{L^{2}(\Omega)}^{2}+C\left\|\nabla V_{\zeta}\right\|_{L^{2}(\Omega)}^{2}+C\left\|V_{\zeta \zeta}\right\|_{L^{2}(\Omega)}^{2} \\
& +C\left(1+\|q\|_{L^{4}(\Omega)}^{8}+\|V\|_{L^{4}(\Omega)}^{8}\right)\left\|q_{\zeta}\right\|_{L^{2}(\Omega)}^{2} \\
& +C\|q\|_{L^{4}(\Omega)}^{8}\left\|V_{\zeta}\right\|_{L^{2}(\Omega)}^{2}+\varepsilon C\left(\left\|\nabla q_{\zeta}\right\|_{L^{2}(\Omega)}^{2}+\left\|q_{\zeta \zeta}\right\|_{L^{2}(\Omega)}^{2}\right)+C(M)\left\|q_{\zeta}\right\|_{L^{2}(\Omega)}^{2} \\
\leq & C(M)+C(M)\left\|q_{\zeta}\right\|_{L^{2}(\Omega)}^{2}+C\left\|\nabla V_{\zeta}\right\|_{L^{2}(\Omega)}^{2}+C\left\|V_{\zeta \zeta}\right\|_{L^{2}(\Omega)}^{2} \\
& +\varepsilon C\left(\left\|\nabla q_{\zeta}\right\|_{L^{2}(\Omega)}^{2}+\left\|q_{\zeta \zeta}\right\|_{L^{2}(\Omega)}^{2}\right) \\
& \\
&
\end{aligned}
$$


By applying the Hardy inequality, we find that

$$
\begin{aligned}
& \left|-\int_{\Omega} \frac{\partial}{\partial \zeta}\left(\frac{1}{\widetilde{p}_{s} \zeta} \int_{0}^{\zeta} \nabla \cdot\left(\widetilde{p_{s}} V\right) d s\right) \tilde{p}_{s} q_{\zeta} d \sigma d \zeta\right| \\
& \quad=\left|\frac{k_{s 2}}{R v_{2}} \int_{S^{2}}\left(\int_{0}^{1} \nabla \cdot\left(\widetilde{p_{s}} V\right) d s\right) q\right|_{\zeta=1} d \sigma+R \int_{\Omega}\left(\frac{1}{\zeta} \int_{0}^{\zeta} \nabla \cdot\left(\widetilde{p}_{s} V\right) d s\right) q_{\zeta \zeta} d \sigma d \zeta \mid \\
& \quad \leq C(M)+C\left\|\left.q\right|_{\zeta=1}\right\|_{L^{2}\left(S^{2}\right)}^{2}+C\left\|\frac{1}{\zeta} \int_{0}^{\zeta} \nabla \cdot\left(\widetilde{p}_{s} V\right) d s\right\|_{L^{2}(\Omega)}^{2}+\varepsilon\left\|q_{\zeta \zeta}\right\|_{L^{2}(\Omega)}^{2} \\
& \quad \leq C(M)+C\left\|\left.q\right|_{\zeta=1}\right\|_{L^{2}\left(S^{2}\right)}^{2}+\varepsilon\left\|q_{\zeta \zeta}\right\|_{L^{2}(\Omega)}^{2},
\end{aligned}
$$

where $\varepsilon>0$ is a small constant, by (4.65),

$$
\begin{aligned}
& \left|\int_{\Omega} \frac{\partial}{\partial \zeta}\left(\delta_{21} \delta_{22} \dot{\zeta} \frac{W(T)}{\zeta}\right) \tilde{p}_{s} q_{\zeta} d \sigma d \zeta\right| \\
& \quad \leq C(M)+C\left\|\left.q\right|_{\zeta=1}\right\|_{L^{2}\left(S^{2}\right)}^{2}+\varepsilon\left\|q_{\zeta \zeta}\right\|_{L^{2}(\Omega)}^{2} .
\end{aligned}
$$

Thanks to $(2.4)_{3}$ and by the boundary conditions, we know that

$$
\begin{aligned}
\left.\left.\nu_{3} \int_{S^{2}} \tilde{p}_{s} q_{\zeta}\right|_{\zeta=1}\left(\left(\frac{g \zeta}{R \widetilde{T}}\right)^{2} q_{\zeta \zeta}\right)\right|_{\zeta=1} d \sigma \\
=\left.\frac{k_{s 3}}{\nu_{3}} \int_{S^{2}} \widetilde{p}_{s} f\left(\left|V_{10}\right|\right)\left(q_{m}^{*}-q\right)\right|_{\zeta=1}\left(\frac{\left.\partial q\right|_{\zeta=1}}{\partial t}+\left.\left(V^{*} \cdot \nabla\right) q\right|_{\zeta=1}-\left.\frac{\mu_{3}}{\widetilde{p}_{s}} \Delta q\right|_{\zeta=1}\right. \\
\left.\quad-\left.\left.\nu_{3} \frac{\partial}{\partial \zeta}\left(\left(\frac{g \zeta}{R \widetilde{T}}\right)^{2}\right)\right|_{\zeta=1} q_{\zeta}\right|_{\zeta=1}\right) d \sigma \\
=-\left.\frac{k_{s 3}}{2 \nu_{3}} \frac{d}{d t} \int_{S^{2}} \widetilde{p}_{s} f\left(\left|V_{10}\right|\right) q^{2}\right|_{\zeta=1} d \sigma+\left.\frac{k_{s 3}}{2 v_{3}} \int_{S^{2}} \frac{d \widetilde{p}_{s}}{d t} f\left(\left|V_{10}\right|\right) q^{2}\right|_{\zeta=1} d \sigma \\
\quad-\left.\frac{k_{s 3} \mu_{3}}{\nu_{3}} \int_{S^{2}}|\nabla q|^{2}\right|_{\zeta=1} d \sigma \\
\quad-\left.\left.\frac{k_{s 3}}{v_{3}} \int_{S^{2}} \widetilde{p}_{s} f\left(\left|V_{10}\right|\right)\right|_{\zeta=1}\left(V^{*} \cdot \nabla\right) q\right|_{\zeta=1} d \sigma \\
\quad-\left.\left.\frac{k_{s 3}^{2}}{v_{3}} \int_{S^{2}} \widetilde{p}_{s} \frac{\partial}{\partial \zeta}\left(\left(\frac{g \zeta}{R \widetilde{T}}\right)^{2}\right)\right|_{\zeta=1} f\left(\left|V_{10}\right|\right) q^{2}\right|_{\zeta=1} d \sigma \\
\quad+\frac{k_{s 3}}{v_{3}} \int_{S^{2}} \widetilde{p}_{s} f\left(\left|V_{10}\right|\right) q_{m}^{*}\left(\frac{\left.\partial q\right|_{\zeta=1}}{\partial t}+\left.\left(V^{*} \cdot \nabla\right) q\right|_{\zeta=1}-\left.\frac{\mu_{3}}{\widetilde{p}_{s}} \Delta q\right|_{\zeta=1}\right. \\
\left.\quad-\left.\left.v_{3} \frac{\partial}{\partial \zeta}\left(\left(\frac{g \zeta}{R \widetilde{T}}\right)^{2}\right)\right|_{\zeta=1} q_{\zeta}\right|_{\zeta=1}\right) d \sigma .
\end{aligned}
$$

By virtue of (4.16) and (4.26), we obtain

$$
\begin{aligned}
& \left|\frac{k_{s 3}}{2 v_{3}} \int_{S^{2}} \frac{d \widetilde{p_{s}}}{d t} f\left(\left|V_{10}\right|\right) q^{2}\right|_{\zeta=1} d \sigma\left|\leq C\left\|\left.q\right|_{\zeta=1}\right\|_{L^{2}\left(S^{2}\right)}^{2},\right. \\
& \left.\left|\frac{k_{s 3}}{v_{3}} \int_{S^{2}} \widetilde{p_{S}} f\left(\left|V_{10}\right|\right) q\right|_{\zeta=1}\left(V^{*} \cdot \nabla\right) q\right|_{\zeta=1} d \sigma \mid
\end{aligned}
$$




$$
\begin{aligned}
&=\left.\left|\frac{k_{s 3}}{\nu_{3}} \int_{S^{2}} f\left(\left|V_{10}\right|\right) q^{2}\right|_{\zeta=1} \nabla \cdot\left(\widetilde{p}_{s} V^{*}\right)\right|_{\zeta=1} d \sigma \mid \\
& \leq\left. C \int_{S^{2}} q^{2}\right|_{\zeta=1}\left(\int_{0}^{1}\left|\nabla \cdot\left(\widetilde{p_{s}} V^{*}\right)\right| d \zeta+\int_{0}^{1}\left|\nabla \cdot\left(\widetilde{p_{s}} V_{\zeta}^{*}\right)\right| d \zeta\right) d \sigma \\
& \leq C(M)+C\left\|\left.q\right|_{\zeta=1}\right\|_{L^{4}\left(S^{2}\right)}^{4}+C\left\|V_{\zeta}\right\|_{L^{2}(\Omega)}^{2}+C\left\|\nabla V_{\zeta}\right\|_{L^{2}(\Omega)}^{2} \\
& \leq C(M)+C\left\|\left.q\right|_{\zeta=1}\right\|_{L^{4}\left(S^{2}\right)}^{4}+C\left\|\nabla V_{\zeta}\right\|_{L^{2}(\Omega)}^{2} \\
&\left.\left|-\frac{k_{s 3}^{2}}{v_{3}} \int_{S^{2}} \widetilde{p_{s}} \frac{\partial}{\partial \zeta}\left(\left(\frac{g \zeta}{R \widetilde{T}}\right)^{2}\right)\right|_{\zeta=} f\left(\left|V_{10}\right|\right) q^{2}\right|_{\zeta=1} d \sigma\left|\leq C\left\|\left.q\right|_{\zeta=1}\right\|_{L^{2}\left(S^{2}\right)^{\prime}}^{2}\right. \\
&\left|\frac{k_{s 3}}{v_{3}} \int_{S^{2}} \widetilde{p}_{s} f\left(\left|V_{10}\right|\right) q_{m}^{*}\left(\left.\left(V^{*} \cdot \nabla\right) q\right|_{\zeta=1}-\left.\frac{\mu_{3}}{\widetilde{p}_{s}} \Delta q\right|_{\zeta=1}-\left.\left.v_{3} \frac{\partial}{\partial \zeta}\left(\left(\frac{g \zeta}{R \widetilde{T}}\right)^{2}\right)\right|_{\zeta=1} q_{\zeta}\right|_{\zeta=1}\right) d \sigma\right| \\
& \quad \leq C(M)+\varepsilon\left\|\left.\nabla q\right|_{\zeta=1}\right\|_{L^{2}\left(S^{2}\right)}^{2}+C\left\|\left.q\right|_{\zeta=1}\right\|_{L^{2}\left(S^{2}\right)}^{2}
\end{aligned}
$$

Using (4.63)-(4.71), we have

$$
\begin{aligned}
& \frac{1}{2} \frac{d}{d t}\left(\int_{\Omega} \widetilde{p}_{s} q_{\zeta}^{2} d \sigma d \zeta+\left.\frac{k_{s 3}}{v_{3}} \int_{S^{2}} \tilde{p}_{s} q^{2}\right|_{\zeta=1} d \sigma\right) \\
& \quad+C \int_{\Omega}\left|\nabla q_{\zeta}\right|^{2} d \sigma d \zeta+C \int_{\Omega} q_{\zeta \zeta}^{2} d \sigma d \zeta+\left.C \int_{S^{2}}|\nabla q|^{2}\right|_{\zeta=1} d \sigma \\
& \leq C(M)+C(M)\left(\int_{\Omega} \widetilde{p}_{s} q_{\zeta}^{2} d \sigma d \zeta+\left.\frac{k_{s 3}}{v_{3}} \int_{S^{2}} \widetilde{p}_{s} q^{2}\right|_{\zeta=1} d \sigma\right) \\
& \quad+\frac{k_{s 3}}{v_{3}} \int_{S^{2}} \widetilde{p}_{s} f\left(\left|V_{10}\right|\right) q_{m}^{*} \frac{\left.\partial q\right|_{\zeta=1}}{\partial t} d \sigma+C\left\|\nabla V_{\zeta}\right\|_{L^{2}(\Omega)}^{2}+C\left\|\left.q\right|_{\zeta=1}\right\|_{L^{4}\left(S^{2}\right)}^{4},
\end{aligned}
$$

which combining with (4.16), (4.26), (4.45) and the Gronwall inequality shows (4.60), where we use the fact that

$$
\begin{aligned}
\mid \frac{k_{s 3}}{v_{3}} & \int_{0}^{t} \int_{S^{2}} \widetilde{p}_{s} f\left(\left|V_{10}\right|\right) q_{m}^{*} \frac{\left.\partial q\right|_{\zeta=1}}{\partial t} d \sigma d \tau \mid \\
= & \left|\frac{k_{s 3}}{v_{3}} \int_{S^{2}} \widetilde{p}_{s} f\left(\left|V_{10}\right|\right) q_{m}^{*} q\right|_{\zeta=1} d \sigma-\left.\frac{k_{s 3}}{v_{3}} \int_{S^{2}} \widetilde{p_{s 0}} f\left(\left|V_{10}\right|\right) q_{m}^{*} q_{0}\right|_{\zeta=1} d \sigma \\
& -\left.\frac{k_{s 3}}{v_{3}} \int_{0}^{t} \int_{S^{2}} \frac{\partial \widetilde{p}_{s}}{\partial t} f\left(\left|V_{10}\right|\right) q_{m}^{*} q\right|_{\zeta=1} d \sigma d \tau \mid \\
\leq & C(M)+\varepsilon\left\|\left.q\right|_{\zeta=1}\right\|_{L^{2}\left(S^{2}\right)^{2}}^{2}
\end{aligned}
$$

Lemma 4.8 Under the assumptions of Theorem 2.1, for any $M>0$ given, the liquid water content $m_{w}$ to the system $(2.4)_{4}$ satisfies

$$
\begin{aligned}
& \int_{\Omega} m_{w \zeta}^{2} d \sigma d \zeta+\int_{0}^{t} \int_{\Omega}\left|\nabla m_{w \zeta}\right|^{2} d \sigma d \zeta d \tau+\int_{0}^{t} \int_{\Omega} m_{w \zeta \zeta}^{2} d \sigma d \zeta d \tau \\
& \quad \leq C(M), \quad t \in[0, M]
\end{aligned}
$$

where $C(M)>0$ denotes a constant dependent of time $M$. 
Proof Taking the derivative with respect to $\zeta$ of $(2.4)_{4}$, we find that

$$
\begin{aligned}
& \frac{\partial m_{w \zeta}}{\partial t}-\mu_{4} \frac{1}{\widetilde{p}_{s}} \Delta m_{w \zeta}-v_{4} \frac{\partial}{\partial \zeta}\left(\left(\frac{g \zeta}{R \widetilde{T}}\right)^{2} m_{w \zeta \zeta}\right) \\
& \quad+\left(\left(V^{*} \cdot \nabla\right) m_{w \zeta}+\dot{\zeta}^{*} m_{w \zeta \zeta}\right)+\left(\left(V_{\zeta}^{*} \cdot \nabla\right) m_{w}-\frac{1}{\widetilde{p}_{s}} \nabla \cdot\left(\widetilde{p_{s}} V^{*}\right) m_{w \zeta}\right) \\
& =v_{4} \frac{\partial}{\partial \zeta}\left(\frac{\partial}{\partial \zeta}\left(\left(\frac{g \zeta}{R \widetilde{T}}\right)^{2}\right) m_{w \zeta}\right)-\frac{\partial}{\partial \zeta}\left(\delta_{21} \delta_{22} \dot{\zeta} \frac{W(T)}{\zeta}\right) \\
& +\frac{\partial}{\partial \zeta}\left(h_{1}\left(\delta_{21} \delta_{22} \dot{\zeta} \frac{W(T)}{\zeta}\right)\right) .
\end{aligned}
$$

Multiplying (4.75) by $\widetilde{p_{s}} m_{w \zeta}$, we find that

$$
\begin{aligned}
\frac{1}{2} & \frac{d}{d t} \int_{\Omega}{\widetilde{p_{s}}}_{m_{w \zeta}}^{2} d \sigma d \zeta+\mu_{4} \int_{\Omega}\left|\nabla m_{w \zeta}\right|^{2} d \sigma d \zeta+v_{4} \int_{\Omega} \widetilde{p}_{s}\left(\frac{g \zeta}{R \widetilde{T}}\right)^{2} m_{w \zeta \zeta}^{2} d \sigma d \zeta \\
= & \int_{\Omega} \frac{d \widetilde{p_{s}}}{d t} m_{w \zeta}^{2} d \sigma d \zeta-\int_{\Omega}\left(\left(V^{*} \cdot \nabla\right) m_{w \zeta}+\dot{\zeta}^{*} m_{w \zeta \zeta}\right) \tilde{p}_{s} m_{w \zeta} d \sigma d \zeta \\
& -\int_{\Omega}\left(\left(V_{\zeta}^{*} \cdot \nabla\right) m_{w}-\frac{1}{\widetilde{p_{s}}} \nabla \cdot\left(\widetilde{p}_{s} V^{*}\right) m_{w \zeta}\right) \tilde{p}_{s} m_{w \zeta} d \sigma d \zeta \\
& +v_{4} \int_{\Omega} \frac{\partial}{\partial \zeta}\left(\frac{\partial}{\partial \zeta}\left(\left(\frac{g \zeta}{R \widetilde{T}}\right)^{2}\right) m_{w \zeta}\right) \tilde{p}_{s} m_{w \zeta} d \sigma d \zeta \\
& +\int_{\Omega} \frac{\partial}{\partial \zeta}\left(h_{1}\left(\delta_{21} \delta_{22} \dot{\zeta} \frac{W(T)}{\zeta}\right)\right) \tilde{p}_{s} m_{w \zeta} d \sigma d \zeta \\
& -\int_{\Omega} \frac{\partial}{\partial \zeta}\left(\delta_{21} \delta_{22} \dot{\zeta} \frac{W(T)}{\zeta}\right) \tilde{p}_{s} m_{w \zeta} d \sigma d \zeta .
\end{aligned}
$$

One can easily check that $\left(V^{*}, \dot{\zeta}^{*}\right)$ satisfies

$$
-\int_{\Omega}\left(\left(V^{*} \cdot \nabla\right) m_{w \zeta}+\dot{\zeta}^{*} m_{w \zeta \zeta}\right) \tilde{p}_{s} m_{w \zeta} d \sigma d \zeta=0
$$

By virtue of (4.16), (4.24)-(4.26), (4.53), the Gagliardo-Nirenberg-Sobolev inequality, the Young inequality and the fact that $V_{\zeta}^{*}=V_{\zeta}$, we deduce that

$$
\begin{aligned}
\mid- & \int_{\Omega}\left(\left(V_{\zeta}^{*} \cdot \nabla\right) m_{w}-\frac{1}{\widetilde{p}_{s}} \nabla \cdot\left(\tilde{p}_{s} V^{*}\right) m_{w \zeta}\right) \tilde{p}_{s} m_{w \zeta} d \sigma d \zeta \mid \\
\leq & C \int_{\Omega}\left(\left(\left|V_{\zeta}\right|+\left|\nabla V_{\zeta}\right|\right)\left|m_{w}\right|\left|m_{w \zeta}\right|+\left|V_{\zeta}\right|\left|m_{w}\right|\left|\nabla m_{w \zeta}\right|+\left|V\left\|m_{w \zeta}\right\| \nabla m_{w \zeta}\right|\right) d \sigma d \zeta \\
\leq & C\left\|V_{\zeta}\right\|_{L^{2}(\Omega)}^{2}+C\left\|\nabla V_{\zeta}\right\|_{L^{2}(\Omega)}^{2}+C\left\|m_{w}\right\|_{L^{4}(\Omega)}^{2}\left\|m_{w \zeta}\right\|_{L^{4}(\Omega)}^{2} \\
& +C\left\|V_{\zeta}\right\|_{L^{4}(\Omega)}^{2}\left\|m_{w}\right\|_{L^{4}(\Omega)}^{2} \\
& +C\|V\|_{L^{4}(\Omega)}^{2}\left\|m_{w \zeta}\right\|_{L^{4}(\Omega)}^{2}+\varepsilon\left\|\nabla m_{w \zeta}\right\|_{L^{2}(\Omega)}^{2} \\
\leq & C\left\|V_{\zeta}\right\|_{L^{2}(\Omega)}^{2}+C\left\|\nabla V_{\zeta}\right\|_{L^{2}(\Omega)}^{2} \\
& +C\left\|m_{w}\right\|_{L^{4}(\Omega)}^{2}\left\|m_{w \zeta}\right\|_{L^{2}(\Omega)}^{\frac{1}{2}}\left(\left\|m_{w \zeta}\right\|_{L^{2}(\Omega)}+\left\|\nabla m_{w \zeta}\right\|_{L^{2}(\Omega)}+\left\|m_{w \zeta \zeta}\right\|_{L^{2}(\Omega)}\right)^{\frac{3}{2}}
\end{aligned}
$$




$$
\begin{aligned}
& +C\left\|V_{\zeta}\right\|_{L^{2}(\Omega)}^{\frac{1}{2}}\left(\left\|V_{\zeta}\right\|_{L^{2}(\Omega)}+\left\|\nabla V_{\zeta}\right\|_{L^{2}(\Omega)}+\left\|V_{\zeta \zeta}\right\|_{L^{2}(\Omega)}\right)^{\frac{3}{2}}\left\|m_{w}\right\|_{L^{4}(\Omega)}^{2} \\
& +C\|V\|_{L^{4}(\Omega)}^{2}\left\|m_{w \zeta}\right\|_{L^{2}(\Omega)}^{\frac{1}{2}}\left(\left\|m_{w \zeta}\right\|_{L^{2}(\Omega)}+\left\|\nabla m_{w \zeta}\right\|_{L^{2}(\Omega)}+\left\|m_{w \zeta \zeta}\right\|_{L^{2}(\Omega)}\right)^{\frac{3}{2}} \\
& +\varepsilon\left\|\nabla m_{w \zeta}\right\|_{L^{2}(\Omega)}^{2} \\
& \leq C\left\|V_{\zeta}\right\|_{L^{2}(\Omega)}^{2}+C\left\|\nabla V_{\zeta}\right\|_{L^{2}(\Omega)}^{2}+C\left\|V_{\zeta \zeta}\right\|_{L^{2}(\Omega)}^{2} \\
& +C\left(1+\left\|m_{w}\right\|_{L^{4}(\Omega)}^{8}+\|V\|_{L^{4}(\Omega)}^{8}\right)\left\|m_{w \zeta}\right\|_{L^{2}(\Omega)}^{2} \\
& +C\left\|m_{w}\right\|_{L^{4}(\Omega)}^{8}\left\|V_{\zeta}\right\|_{L^{2}(\Omega)}^{2}+\varepsilon C\left(\left\|\nabla m_{w \zeta}\right\|_{L^{2}(\Omega)}^{2}+\left\|m_{w \zeta \zeta}\right\|_{L^{2}(\Omega)}^{2}\right) \\
& \leq C(M)+C(M)\left\|m_{w \zeta}\right\|_{L^{2}(\Omega)}^{2}+C\left\|\nabla V_{\zeta}\right\|_{L^{2}(\Omega)}^{2}+C\left\|V_{\zeta \zeta}\right\|_{L^{2}(\Omega)}^{2} \\
& \quad+\varepsilon C\left(\left\|\nabla m_{w \zeta}\right\|_{L^{2}(\Omega)}^{2}+\left\|m_{w \zeta \zeta}\right\|_{L^{2}(\Omega)}^{2}\right)
\end{aligned}
$$

where $\varepsilon>0$ is a small constant.

Using (4.16), the Young inequality and the Hardy inequality, we find that

$$
\begin{aligned}
& \left|-\int_{\Omega} \frac{\partial}{\partial \zeta}\left(\delta_{21} \delta_{22} \frac{1}{\widetilde{p}_{s} \zeta} \int_{0}^{\zeta} \nabla \cdot\left(\widetilde{p}_{s} V\right) d s\right) \widetilde{p}_{s} m_{w \zeta} d \sigma d \zeta\right| \\
& \quad=\left|\int_{\Omega} \delta_{21} \delta_{22}\left(\frac{1}{\zeta} \int_{0}^{\zeta} \nabla \cdot\left(\widetilde{p}_{s} V\right) d s\right) m_{w \zeta \zeta} d \sigma d \zeta\right| \\
& \quad \leq C\left\|\frac{1}{\zeta} \int_{0}^{\zeta} \nabla \cdot\left(\widetilde{p}_{s} V\right) d s\right\|_{L^{2}(\Omega)}^{2}+\varepsilon\left\|m_{w \zeta \zeta}\right\|_{L^{2}(\Omega)}^{2} \\
& \quad \leq C(M)+\varepsilon\left\|m_{w \zeta \zeta}\right\|_{L^{2}(\Omega)}^{2}, \\
& \left|\int_{\Omega} \nabla \cdot\left(\widetilde{p_{s}} \bar{V}\right) \delta_{21} \delta_{22} W(T) m_{w \zeta \zeta} d \sigma d \zeta\right| \\
& \quad \leq C(M)+\varepsilon\left\|m_{w \zeta \zeta}\right\|_{L^{2}(\Omega)}^{2} .
\end{aligned}
$$

Then we get

$$
\left\|-\int_{\Omega} \frac{\partial}{\partial \zeta}\left(\delta_{21} \delta_{22} \dot{\zeta} \frac{W(T)}{\zeta}\right) \widetilde{p}_{s} m_{w \zeta} d \sigma d \zeta\right\| \leq C(M)+\varepsilon\left\|m_{w \zeta \zeta}\right\|_{L^{2}(\Omega)}^{2}
$$

where $\varepsilon>0$ is a small constant.

Applying (4.77)-(4.81), we deduce that

$$
\begin{aligned}
& \frac{d}{d t} \int_{\Omega} \widetilde{p}_{s} m_{w \zeta}^{2} d \sigma d \zeta+C \int_{\Omega}\left|\nabla m_{w \zeta}\right|^{2} d \sigma d \zeta+C \int_{\Omega} m_{w \zeta \zeta}^{2} d \sigma d \zeta \\
& \quad \leq C(M)+C(M)\left\|m_{w \zeta}\right\|_{L^{2}(\Omega)}^{2}+C\left\|\nabla V_{\zeta}\right\|_{L^{2}(\Omega)}^{2}+C\left\|V_{\zeta \zeta}\right\|_{L^{2}(\Omega)}^{2} \\
& \quad+\varepsilon C\left(\left\|\nabla m_{w \zeta}\right\|_{L^{2}(\Omega)}^{2}+\left\|m_{w \zeta \zeta}\right\|_{L^{2}(\Omega)}^{2}\right)
\end{aligned}
$$

which combining with (4.16), Lemma 4.3 and the Gronwall inequality gives (4.74). 
Lemma 4.9 Under the assumptions of Theorem 2.1, for any $M>0$ given, the specific humidity $q$ to the system $(2.4)_{3}$ satisfies

$$
\begin{aligned}
& \int_{\Omega}|\nabla q|^{2} d \sigma d \zeta+\int_{0}^{t} \int_{\Omega}|\Delta q|^{2} d \sigma d \zeta d \tau+\int_{0}^{t} \int_{\Omega}\left|\nabla q_{\zeta}\right|^{2} d \sigma d \zeta d \tau \\
& +\left.\int_{0}^{t} \int_{S^{2}}|\nabla q|^{2}\right|_{\zeta=1} d \sigma d \tau \leq C(M), \quad t \in[0, M],
\end{aligned}
$$

where $C(M)>0$ denotes a constant dependent on time $M$.

Proof Taking the inner product of $(2.4)_{3}$ with $\Delta q$, we find that

$$
\begin{aligned}
& \frac{1}{2} \frac{d}{d t} \int_{\Omega}|\nabla q|^{2} d \sigma d \zeta+\mu_{3} \int_{\Omega} \frac{1}{\widetilde{p_{s}}}|\Delta q|^{2} d \sigma d \zeta+\nu_{3} \int_{\Omega}\left(\frac{g \zeta}{R \widetilde{T}}\right)^{2}\left|\nabla q_{\zeta}\right|^{2} d \sigma d \zeta \\
& \quad+\left.\nu_{3} k_{s 3} \int_{S^{2}}\left(\left(\frac{g \zeta}{R \widetilde{T}}\right)^{2} f\left(\left|V_{10}\right|\right)|\nabla q|^{2}\right)\right|_{\zeta=1} d \sigma \\
& =\int_{\Omega}\left(V^{*} \cdot \nabla\right) q \Delta q d \sigma d \zeta+\int_{\Omega} \dot{\zeta}^{*} q_{\zeta} \Delta q d \sigma d \zeta \\
& \quad+\int_{\Omega}\left(\delta_{21} \delta_{22} \frac{\dot{\zeta}}{\zeta} W(T)\right) \widetilde{p}_{s} \Delta q d \sigma d \zeta \\
& \quad+\left.\int_{S^{2}}\left(\frac{g \zeta}{R \widetilde{T}} f\left(\left|V_{10}\right|\right) q_{m}^{*} \Delta q\right)\right|_{\zeta=1} d \sigma .
\end{aligned}
$$

Thanks to (4.16) and (4.25), we get

$$
\begin{aligned}
& \left|\int_{\Omega}\left(V^{*} \cdot \nabla\right) q \Delta q d \sigma d \zeta\right| \\
& \quad \leq C\left\|V^{*} \nabla q\right\|_{L^{2}(\Omega)}^{2}+\varepsilon\|\Delta q\|_{L^{2}(\Omega)}^{2} \\
& \quad \leq C\|V\|_{L^{4}(\Omega)}^{2}\|\nabla q\|_{L^{4}(\Omega)}^{2}+\varepsilon\|\Delta q\|_{L^{2}(\Omega)}^{2} \\
& \quad \leq C\|V\|_{L^{4}(\Omega)}^{2}\|\nabla q\|_{L^{2}(\Omega)}^{\frac{1}{2}}\left(\|\nabla q\|_{L^{2}(\Omega)}+\|\Delta q\|_{L^{2}(\Omega)}+\left\|\nabla q_{\zeta}\right\|_{L^{2}(\Omega)}\right)^{\frac{3}{2}}+\varepsilon\|\Delta q\|_{L^{2}(\Omega)}^{2} \\
& \quad \leq C\left(1+\|V\|_{L^{4}(\Omega)}^{8}\right)\|\nabla q\|_{L^{2}(\Omega)}^{2}+\varepsilon C\|\Delta q\|_{L^{2}(\Omega)}^{2}+\varepsilon\left\|\nabla q_{\zeta}\right\|_{L^{2}(\Omega)}^{2} \\
& \quad \leq C(M)\|\nabla q\|_{L^{2}(\Omega)}^{2}+\varepsilon C\|\Delta q\|_{L^{2}(\Omega)}^{2}+\varepsilon\left\|\nabla q_{\zeta}\right\|_{L^{2}(\Omega)}^{2}
\end{aligned}
$$

where $\varepsilon>0$ is a small constant.

By (4.16), (4.28) and (4.60), we infer

$$
\begin{aligned}
& \left|\int_{\Omega} \dot{\zeta}^{*} q_{\zeta} \Delta q d \sigma d \zeta\right| \\
& \quad \leq C \int_{S^{2}}\left(\int_{0}^{1}\left(\int_{0}^{\zeta}\left|\nabla \cdot\left(\widetilde{p}_{s} V^{*}\right)\right| d s+\left|\frac{\partial \widetilde{p_{s}}}{\partial t}\right|\right)\left|q_{\zeta}\right||\Delta q| d \zeta\right) d \sigma \\
& \quad \leq C \int_{S^{2}}\left(\int_{0}^{1}\left|\nabla \cdot\left(\widetilde{p}_{s} V^{*}\right)\right|^{2} d \zeta+C(M)\right)\left(\int_{0}^{1}\left|q_{\zeta}\right|^{2} d \zeta\right) d \sigma+\varepsilon\|\Delta q\|_{L^{2}(\Omega)}^{2} \\
& \quad \leq C\left(\int_{S^{2}}\left(\int_{0}^{1}\left|\nabla \cdot\left(\widetilde{p}_{s} V^{*}\right)\right|^{2} d \zeta+C(M)\right)^{2} d \sigma\right)^{\frac{1}{2}}\left(\int_{S^{2}}\left(\int_{0}^{1}\left|q_{\zeta}\right|^{2} d \zeta\right)^{2} d \sigma\right)^{\frac{1}{2}}
\end{aligned}
$$




$$
\begin{aligned}
& +\varepsilon\|\Delta q\|_{L^{2}(\Omega)}^{2} \\
\leq & C\left(\int_{0}^{1}\left(\int_{S^{2}}\left|\nabla \cdot\left(\widetilde{p}_{s} V^{*}\right)\right|^{4} d \sigma+C(M)\right)^{\frac{1}{2}} d \zeta\right)\left(\int_{0}^{1}\left(\int_{S^{2}}\left|q_{\zeta}\right|^{4} d \sigma\right)^{\frac{1}{2}} d \zeta\right) \\
& +\varepsilon\|\Delta q\|_{L^{2}(\Omega)}^{2} \\
\leq & C\left(\int_{0}^{1}\left\|\nabla \cdot\left(\widetilde{p}_{s} V^{*}\right)\right\|_{L^{2}\left(S^{2}\right)}\left\|\nabla \cdot\left(\widetilde{p}_{s} V^{*}\right)\right\|_{H^{1}\left(S^{2}\right)} d \zeta+C(M)\right) \\
& \times\left(\int_{0}^{1}\left\|q_{\zeta}\right\|_{L^{2}\left(S^{2}\right)}\left\|q_{\zeta}\right\|_{H^{1}\left(S^{2}\right)} d \zeta\right)+\varepsilon\|\Delta q\|_{L^{2}(\Omega)}^{2} \\
\leq & C\left(\left\|\nabla \cdot\left(\widetilde{p_{s}} V\right)\right\|_{L^{2}(\Omega)}+C(M)\right)\left(\left\|\nabla \cdot\left(\widetilde{p}_{s} V\right)\right\|_{L^{2}(\Omega)}+\left\|\Delta\left(\widetilde{p_{s}} V\right)\right\|_{L^{2}(\Omega)}\right) \\
& \times\left\|q_{\zeta}\right\|_{L^{2}(\Omega)}\left(\left\|q_{\zeta}\right\|_{L^{2}(\Omega)}+\left\|\nabla q_{\zeta}\right\|_{L^{2}(\Omega)}\right)+\varepsilon\|\Delta q\|_{L^{2}(\Omega)}^{2} \\
\leq & C(M)+C(M)\|\Delta V\|_{L^{2}(\Omega)}^{2}+\varepsilon\|\Delta q\|_{L^{2}(\Omega)}^{2}+\varepsilon\left\|\nabla q_{\zeta}\right\|_{L^{2}(\Omega)}^{2}
\end{aligned}
$$

where $\varepsilon>0$ is a small constant.

Applying (4.16), (4.60), the Hardy inequality and the Young inequality, we also obtain

$$
\begin{aligned}
& \left|R \int_{\Omega} \frac{1}{\widetilde{\tilde{p}_{s} \zeta}}\left(\int_{0}^{\zeta} \nabla \cdot\left(\widetilde{p_{s}} V\right) d s\right) \Delta q d \sigma d \zeta\right| \\
& \quad \leq C\left\|\nabla \cdot\left(\widetilde{p_{s}} V\right) d s\right\|_{L^{2}(\Omega)}\|\Delta q\|_{L^{2}(\Omega)} \leq C(M)+\varepsilon\|\Delta q\|_{L^{2}(\Omega)}^{2}, \\
& \left|\int_{\Omega} \frac{1}{\widetilde{\widetilde{p}}_{s}} \nabla \cdot\left(\widetilde{p_{s}} \bar{V}\right) \Delta q d \sigma d \zeta\right| \leq C(M)+\varepsilon\|\Delta q\|_{L^{2}(\Omega)}^{2}, \\
& \left\|\left.\int_{S^{2}} \frac{g \zeta}{R \widetilde{T}} f\left(\left|V_{10}\right|\right) q_{m}^{*} \Delta q\right|_{\zeta=1} d \sigma\right\| \leq C(M)+\varepsilon\left\|\left.\nabla q\right|_{\zeta=1}\right\|_{L^{2}\left(s^{2}\right)^{\prime}}^{2} \\
& \left|\int_{\Omega} \delta_{21} \delta_{22} \frac{\dot{\zeta}}{\zeta} W(T) \widetilde{p_{s}} \Delta q d \sigma d \zeta\right| \leq C(M)+\varepsilon\|\Delta q\|_{L^{2}(\Omega)}^{2},
\end{aligned}
$$

where $\varepsilon>0$ is a small constant.

By (4.85)-(4.90), we obtain

$$
\begin{aligned}
& \frac{d}{d t} \int_{\Omega}|\nabla q|^{2} d \sigma d \zeta+C \int_{\Omega}|\Delta q|^{2} d \sigma d \zeta+C \int_{\Omega}\left|\nabla q_{\zeta}\right|^{2} d \sigma d \zeta \\
& \quad+\left.C \int_{S^{2}}|\nabla q|^{2}\right|_{\zeta=1} d \sigma \\
& \quad \leq C(M)+C(M)\|\nabla q\|_{L^{2}(\Omega)}^{2}+C\|\Delta V\|_{L^{2}(\Omega)}^{2},
\end{aligned}
$$

which combining with (4.16), (4.28), (4.60) and the Gronwall inequality shows (4.83).

Lemma 4.10 Under the assumptions of Theorem 2.1, for any $M>0$ given, the liquid water content $m_{w}$ to the system $(2.4)_{4}$ satisfies

$$
\begin{gathered}
\int_{\Omega}\left|\nabla m_{w}\right|^{2} d \sigma d \zeta+\int_{0}^{t} \int_{\Omega}\left|\Delta m_{w}\right|^{2} d \sigma d \zeta d \tau \\
+\int_{0}^{t} \int_{\Omega}\left|\nabla m_{w \zeta}\right|^{2} d \sigma d \zeta d \tau
\end{gathered}
$$


Lien and Ma Boundary Value Problems

(2020) 2020:103

Page 26 of 34

$$
\leq C(M), \quad t \in[0, M]
$$

where $C(M)>0$ denotes a constant dependent of time $M$.

Proof Taking the inner product of $(2.4)_{4}$ with $\Delta m_{w}$, we know that

$$
\begin{aligned}
& \frac{1}{2} \frac{d}{d t} \int_{\Omega}\left|\nabla m_{w}\right|^{2} d \sigma d \zeta+\mu_{4} \int_{\Omega} \frac{1}{\widetilde{p}_{s}}\left|\Delta m_{w}\right|^{2} d \sigma d \zeta+v_{4} \int_{\Omega}\left(\frac{g \zeta}{R \widetilde{T}}\right)^{2}\left|\nabla m_{w \zeta}\right|^{2} d \sigma d \zeta \\
& \quad=\int_{\Omega}\left(V^{*} \cdot \nabla\right) m_{w} \Delta m_{w} d \sigma d \zeta+\int_{\Omega} \dot{\zeta}^{*} m_{w \zeta} \Delta m_{w} d \sigma d \zeta \\
& \quad+\int_{\Omega} h_{1}\left(\delta_{21} \delta_{22} \dot{\zeta} \frac{W(T)}{\zeta}\right) \tilde{p}_{s} \Delta m_{w} d \sigma d \zeta \\
& \quad-\int_{\Omega} \delta_{21} \delta_{22} \dot{\zeta} \frac{W(T)}{\zeta} \widetilde{p}_{s} \Delta m_{w} d \sigma d \zeta .
\end{aligned}
$$

By (4.16) and (4.25), we obtain

$$
\begin{aligned}
& \left|\int_{\Omega}\left(V^{*} \cdot \nabla\right) m_{w} \Delta m_{w} d \sigma d \zeta\right| \\
& \quad \leq C \int_{\Omega}\left|V^{*}\right|^{2}\left|\nabla m_{w}\right|^{2} d \sigma d \zeta+\varepsilon\left\|\Delta m_{w}\right\|_{L^{2}(\Omega)}^{2} \\
& \quad \leq C\|V\|_{L^{4}(\Omega)}^{2}\left\|\nabla m_{w}\right\|_{L^{4}(\Omega)}^{2}+\varepsilon\left\|\Delta m_{w}\right\|_{L^{2}(\Omega)}^{2} \\
& \quad \leq C\|V\|_{L^{4}(\Omega)}^{2}\left\|\nabla m_{w}\right\|_{L^{2}(\Omega)}^{\frac{1}{2}}\left(\left\|\nabla m_{w}\right\|_{L^{2}(\Omega)}+\left\|\Delta m_{w}\right\|_{L^{2}(\Omega)}+\left\|\nabla m_{w \zeta}\right\|_{L^{2}(\Omega)}\right)^{\frac{3}{2}} \\
& \quad+\varepsilon\left\|\Delta m_{w}\right\|_{L^{2}(\Omega)}^{2} \\
& \quad \leq C\left(1+\|V\|_{L^{4}(\Omega)}^{8}\right)\left\|\nabla m_{w}\right\|_{L^{2}(\Omega)}^{2}+\varepsilon C\left\|\Delta m_{w}\right\|_{L^{2}(\Omega)}^{2}+\varepsilon\left\|\nabla m_{w}\right\|_{L^{2}(\Omega)}^{2} \\
& \leq C(M)\left\|\nabla m_{w}\right\|_{L^{2}(\Omega)}^{2}+\varepsilon C\left\|\Delta m_{w}\right\|_{L^{2}(\Omega)}^{2}+\varepsilon\left\|\nabla m_{w}\right\|_{L^{2}(\Omega)}^{2},
\end{aligned}
$$

where $\varepsilon>0$ is a small constant.

Thanks to (4.16), (4.28) and (4.74), we have

$$
\begin{aligned}
& \left|\int_{\Omega} \dot{\zeta}^{*} m_{w \zeta} \Delta m_{w} d \sigma d \zeta\right| \\
& \quad \leq C \int_{S^{2}}\left(\int_{0}^{1}\left(\int_{0}^{\zeta}\left|\nabla \cdot\left(\tilde{p}_{s} V^{*}\right)\right| d s+\left|\frac{\partial \widetilde{p_{s}}}{\partial t}\right|\right)\left|m_{w \zeta}\right|\left|\Delta m_{w}\right| d \zeta\right) d \sigma \\
& \leq C \int_{S^{2}}\left(\int_{0}^{1}\left|\nabla \cdot\left(\widetilde{p_{s}} V^{*}\right)\right|^{2} d \zeta+C(M)\right)\left(\int_{0}^{1}\left|m_{w \zeta}\right|^{2} d \zeta\right) d \sigma+\varepsilon\left\|\Delta m_{w}\right\|_{L^{2}(\Omega)}^{2} \\
& \leq C\left(\int_{S^{2}}\left(\int_{0}^{1}\left|\nabla \cdot\left(\tilde{p}_{s} V^{*}\right)\right|^{2} d \zeta+C(M)\right)^{2} d \sigma\right)^{\frac{1}{2}}\left(\int_{S^{2}}\left(\int_{0}^{1}\left|m_{w \zeta}\right|^{2} d \zeta\right)^{2} d \sigma\right)^{\frac{1}{2}} \\
& \quad+\varepsilon\left\|\Delta m_{w}\right\|_{L^{2}(\Omega)}^{2} \\
& \quad \leq\left(\int_{0}^{1}\left(\int_{S^{2}}\left|\nabla \cdot\left(\tilde{p}_{s} V^{*}\right)\right|^{4} d \sigma+C(M)\right)^{\frac{1}{2}} d \zeta\right)\left(\int_{0}^{1}\left(\int_{S^{2}}\left|m_{w \zeta}\right|^{4} d \sigma\right)^{\frac{1}{2}} d \zeta\right) \\
& \quad+\varepsilon\left\|\Delta m_{w}\right\|_{L^{2}(\Omega)}^{2}
\end{aligned}
$$




$$
\begin{aligned}
\leq & C\left(\int_{0}^{1}\left\|\nabla \cdot\left(\widetilde{p}_{s} V^{*}\right)\right\|_{L^{2}\left(S^{2}\right)}\left\|\nabla \cdot\left(\widetilde{p}_{s} V^{*}\right)\right\|_{H^{1}\left(S^{2}\right)} d \zeta+C(M)\right) \\
& \times\left(\int_{0}^{1}\left\|m_{w \zeta}\right\|_{L^{2}\left(S^{2}\right)}\left\|m_{w \zeta}\right\|_{H^{1}\left(S^{2}\right)} d \zeta\right)+\varepsilon\left\|\Delta m_{w}\right\|_{L^{2}(\Omega)}^{2} \\
\leq & C\left\|\nabla \cdot\left(\widetilde{p}_{s} V\right)\right\|_{L^{2}(\Omega)}\left(\left\|\nabla \cdot\left(\widetilde{p_{s}} V\right)\right\|_{L^{2}(\Omega)}+\left\|\Delta\left(\widetilde{p_{s}} V\right)\right\|_{L^{2}(\Omega)}\right) \\
& \times\left\|m_{w \zeta}\right\|_{L^{2}(\Omega)}\left(\left\|m_{w \zeta}\right\|_{L^{2}(\Omega)}+\left\|\nabla m_{w \zeta}\right\|_{L^{2}(\Omega)}\right)+\varepsilon\left\|\Delta m_{w}\right\|_{L^{2}(\Omega)}^{2} \\
\leq & C(M)+C(M)\|\Delta V\|_{L^{2}(\Omega)}^{2}+\varepsilon\left\|\Delta m_{w}\right\|_{L^{2}(\Omega)}^{2}+\varepsilon\left\|\nabla m_{w \zeta}\right\|_{L^{2}(\Omega)}^{2},
\end{aligned}
$$

where $\varepsilon>0$ is a small constant.

By (4.28), the Cauchy-Schwarz inequality, the Hardy inequality and the Young inequality, we get

$$
\begin{aligned}
& \| \int_{\Omega} h_{1}\left(\delta_{21} \delta_{22} \dot{\zeta} \frac{W(T)}{\zeta}\right) \widetilde{p}_{s} \Delta m_{w} d \sigma d \zeta \\
& \quad-\int_{\Omega} \delta_{21} \delta_{22} \dot{\zeta} \frac{W(T)}{\zeta} \widetilde{p}_{s} \Delta m_{w} d \sigma d \zeta \| \\
& \leq C\left|\int_{\Omega} \delta_{21} \delta_{22} \nabla \cdot\left(\widetilde{p_{s}} \bar{V}\right) W(T) \Delta m_{w} d \sigma d \zeta\right| \\
& \quad+C\left|\int_{\Omega}\left(\delta_{21} \delta_{22} \frac{1}{\zeta} \int_{0}^{\zeta} \nabla \cdot\left(\widetilde{p_{s}} V\right) d s\right) W(T) \Delta m_{w} d \sigma d \zeta\right| \\
& \quad+C\left|\int_{\Omega} \Delta m_{w} d \sigma d \zeta\right| \\
& \leq C(M)+\varepsilon\left\|\Delta m_{w}\right\|_{L^{2}(\Omega)}^{2}
\end{aligned}
$$

where $C(M)>0$ denotes a constant dependent of time $M$ and $\varepsilon>0$ is a small constant such that

$$
\begin{aligned}
& \frac{d}{d t} \int_{\Omega}\left|\nabla m_{w}\right|^{2} d \sigma d \zeta+C \int_{\Omega}\left|\Delta m_{w}\right|^{2} d \sigma d \zeta+C \int_{\Omega}\left|\nabla m_{w \zeta}\right|^{2} d \sigma d \zeta \\
& \quad \leq C(M)+C(M)\|\Delta V\|_{L^{2}(\Omega)}^{2}+C(M)\left\|\nabla m_{w}\right\|_{L^{2}(\Omega)}^{2},
\end{aligned}
$$

thanks to the Gronwall inequality, we deduce (4.92).

\section{Conclusions}

\subsection{Proof of Theorem 2.1}

Proof By (4.16), (4.26)-(4.29), (4.60), (4.74), (4.83), (4.92) and the proof of the short time existence in Refs. [10, 23], we can extend the strong solution $U$ to the system (2.4) beyond $M_{*}$, contradicting the fact that $M_{*}$ is a finite maximal time of existence. This contradiction means that $M_{*}=+\infty$; then we get the global existence of strong solution.

Next, we will show the uniqueness of global strong solution as follows: let $\left(V_{1}, T_{1}^{\prime}, q_{1}\right.$, $\left.m_{w 1}\right)$ and $\left(V_{2}, T_{2}^{\prime}, q_{2}, m_{w 2}\right)$ be two strong solutions of system (2.4) on the time interval $[0, M]$ with the initial data $\left(V_{01}, T_{01}^{\prime}, q_{01}, m_{w 01}\right)$ and $\left(V_{02}, T_{02}^{\prime}, q_{02}, m_{w 02}\right)$, respectively. Define $V=V_{1}-V_{2}, T^{\prime}=T_{1}^{\prime}-T_{2}^{\prime}, q=q_{1}-q_{2}, m_{w}=m_{w 1}-m_{w 2}$. Then $V, T^{\prime}, q, m_{w}$ satisfy the 
following system:

$$
\begin{aligned}
& \int \frac{\partial V}{\partial t}-\frac{\mu_{1}}{\widetilde{p_{s}}} \Delta V-v_{1} \frac{\partial}{\partial \zeta}\left(\left(\frac{g \zeta}{R T}\right)^{2} \frac{\partial V}{\partial \zeta}\right)+\nabla_{V_{1}^{*}} V+\nabla_{V^{*}} V_{2} \\
& -\frac{1}{\widetilde{p}_{s}}\left(\int_{0}^{\zeta} \nabla \cdot\left(\widetilde{p}_{s} V_{1}^{*}\right) d s+\frac{\partial \widetilde{p}_{s}}{\partial t} \zeta\right) \frac{\partial V}{\partial \zeta} \\
& -\frac{1}{\widetilde{p}_{s}}\left(\int_{0}^{\zeta} \nabla \cdot\left(\widetilde{p_{s}} V^{*}\right) d s\right) \frac{\partial V_{2}}{\partial \zeta}+2 \omega \cos \theta\left(\begin{array}{cc}
0 & 1 \\
-1 & 0
\end{array}\right) V+R \int_{\zeta}^{1} \frac{\nabla T^{\prime}(s)}{s} d s+R T^{\prime} \frac{\nabla \widetilde{p_{s}}}{\widetilde{p_{s}}} \\
& -\nabla\left(\frac{R \widetilde{T}_{s}}{\widetilde{p}_{s}} \int_{0}^{t} \nabla \cdot\left(\widetilde{p_{s}} \bar{V}\right) d \tau\right)=0 \\
& \frac{R}{c_{0}^{2}} \frac{\partial T^{\prime}}{\partial t}-\frac{R \mu_{2}}{c_{p} c_{0}^{2}} \frac{1}{\widetilde{p}_{s}} \Delta T^{\prime}-\frac{R \nu_{2}}{c_{p} c_{0}^{2}} \frac{\partial}{\partial \zeta}\left(\left(\frac{g \zeta}{R T}\right)^{2} \frac{\partial T^{\prime}}{\partial \zeta}\right)+\frac{R}{c_{0}^{2}}\left(V_{1}^{*} \cdot \nabla\right) T^{\prime}+\frac{R}{c_{0}^{2}}\left(V^{*} \cdot \nabla\right) T_{2}^{\prime} \\
& -\frac{R}{c_{0}^{2}} \frac{1}{\widetilde{p}_{s}}\left(\int_{0}^{\zeta} \nabla \cdot\left(\widetilde{p_{s}} V_{1}^{*}\right) d s\right) \frac{\partial T^{\prime}}{\partial \zeta}-\frac{R}{c_{0}^{2}} \frac{1}{\widetilde{p_{s}}}\left(\int_{0}^{\zeta} \nabla \cdot\left(\widetilde{p_{s}} V^{*}\right) d s\right) \frac{\partial T_{2}^{\prime}}{\partial \zeta} \\
& +\frac{R}{\widetilde{p_{s} \zeta}} \int_{0}^{\zeta} \nabla \cdot\left(\widetilde{p_{s}} V\right) d s-\frac{R}{\widetilde{p_{s}}} \nabla \widetilde{p_{s}} \cdot V=0, \\
& \frac{\partial q}{\partial t}-\mu_{3} \frac{1}{\widetilde{p}_{s}} \Delta q-v_{3} \frac{\partial}{\partial \zeta}\left(\left(\frac{g \zeta}{R T}\right)^{2} \frac{\partial q}{\partial \zeta}\right)+\left(V_{1}^{*} \cdot \nabla\right) q+\left(V^{*} \cdot \nabla\right) q_{2} \\
& -\frac{1}{\widetilde{p_{s}}}\left(\int_{0}^{\zeta} \nabla \cdot\left(\widetilde{p_{s}} V_{1}^{*}\right) d s\right) \frac{\partial q}{\partial \zeta}-\frac{1}{\widetilde{p_{s}}}\left(\int_{0}^{\zeta} \nabla \cdot\left(\widetilde{p_{s}} V^{*}\right) d s\right) \frac{\partial q_{2}}{\partial \zeta} \\
& -\frac{\delta_{21} \delta_{22}}{\widetilde{p}_{s} \zeta} \int_{0}^{\zeta} \nabla \cdot\left(\tilde{p}_{s} V\right) d s W(T)+\frac{\delta_{21} \delta_{22}}{\widetilde{p}_{s}} \nabla \cdot\left(\widetilde{p_{s}} \bar{V}\right) W(T)=0, \\
& \frac{\partial m_{w}}{\partial t}-\mu_{4} \frac{1}{\widetilde{p_{s}}} \Delta m_{w}-v_{4} \frac{\partial}{\partial \zeta}\left(\left(\frac{g \zeta}{R T}\right)^{2} \frac{\partial m_{w}}{\partial \zeta}\right)+\left(V_{1}^{*} \cdot \nabla\right) m_{w}+\left(V^{*} \cdot \nabla\right) q_{2} \\
& -\frac{1}{\widetilde{p_{s}}}\left(\int_{0}^{\zeta} \nabla \cdot\left(\widetilde{p_{s}} V_{1}^{*}\right) d s\right) \frac{\partial m_{w}}{\partial \zeta}-\frac{1}{\widetilde{p_{s}}}\left(\int_{0}^{\zeta} \nabla \cdot\left(\widetilde{p_{s}} V^{*}\right) d s\right) \frac{\partial m_{w 2}}{\partial \zeta} \\
& -\frac{\delta_{21} \delta_{22}}{\widetilde{p}_{s} \zeta} \int_{0}^{\zeta} \nabla \cdot\left(\tilde{p}_{s} V\right) d s W(T)+\frac{\delta_{21} \delta_{22}}{\widetilde{p}_{s}} \nabla \cdot\left(\tilde{p}_{s} \bar{V}\right) W(T) \\
& -h_{1}\left(\frac{\delta_{21} \delta_{22}}{\widetilde{p}_{s} \zeta} \int_{0}^{\zeta} \nabla \cdot\left(\widetilde{p_{s}} V\right) d s W(T)-\frac{\delta_{21} \delta_{22}}{\widetilde{p}_{s}} \nabla \cdot\left(\widetilde{p_{s}} \bar{V}\right) W(T)\right)=0,
\end{aligned}
$$

with the initial data and boundary conditions as follows:

$$
\left\{\begin{array}{l}
\left(\left.V\right|_{t=0},\left.T^{\prime}\right|_{t=0},\left.q\right|_{t=0},\left.m_{w}\right|_{t=0}\right) \\
\quad=\left(V_{01}-V_{02}, T_{01}^{\prime}-T_{02}^{\prime}, q_{01}-q_{02}, m_{w 01}-m_{w 02}\right) \\
\left(V, T^{\prime}, q, m_{w}\right)(\theta, \lambda, p) \\
\quad=\left(V, T^{\prime}, q, m_{w}\right)(\theta+\pi, \lambda, \zeta)=\left(V, T^{\prime}, q, m_{w}\right)(\theta, \lambda+2 \pi, \zeta), \\
\left.\frac{\partial V}{\partial \zeta}\right|_{\zeta=0}=0,\left.\quad \frac{\partial T^{\prime}}{\partial \zeta}\right|_{\zeta=0}=0,\left.\quad \frac{\partial q}{\partial \zeta}\right|_{\zeta=0}=0,\left.a \frac{\partial m_{w}}{\partial \zeta}\right|_{\zeta=0}=0, \\
\left.\left(v_{1} \frac{\partial V}{\partial \zeta}+k_{s 1}\left(f\left(\left|V_{1}\right|\right) V_{1}-f\left(\left|V_{2}\right|\right) V_{2}\right)\right)\right|_{\zeta=1}=0, \\
\left.\left(v_{2} \frac{\partial T^{\prime}}{\partial \zeta}+k_{s 2} T^{\prime}\right)\right|_{\zeta=1}=0,\left.\quad\left(v_{3} \frac{\partial q}{\partial \zeta}+k_{s 3} f\left(\left|V_{10}\right|\right) q\right)\right|_{\zeta=1}=0,\left.\quad \frac{\partial m_{w}}{\partial \zeta}\right|_{\zeta=1}=0
\end{array}\right.
$$

Note that, from [17], we can find that

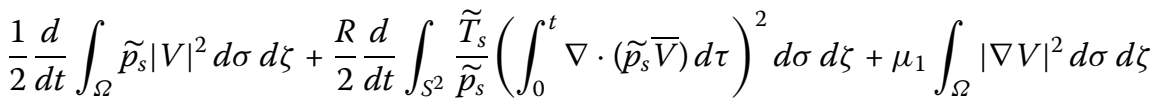

$$
\begin{aligned}
& +v_{1} \int_{\Omega} \widetilde{p_{s}}\left(\frac{g \zeta}{R \widetilde{T}}\right)^{2}\left|\frac{\partial V}{\partial \zeta}\right|^{2} d \sigma d \zeta \\
& +\left.k_{s 1} \int_{S^{2}} \widetilde{p_{s}}\left(\left(\frac{g \zeta}{R \tilde{T}}\right)^{2}\left(f\left(\left|V_{1}\right|\right) V_{1}-f\left(\left|V_{2}\right|\right) V_{2}\right) \cdot\left(V_{1}-V_{2}\right)\right)\right|_{\zeta=1} d \sigma \\
& \leq C(M)\left(1+\left\|\nabla V_{2 \zeta}\right\|_{L^{2}(\Omega)}^{2}\right)\|V\|_{L^{2}(\Omega)}^{2}+C\left\|T^{\prime}\right\|_{L^{2}(\Omega)}^{2} \\
& +R \int_{\Omega} T^{\prime}\left(\frac{1}{\zeta} \int_{0}^{\zeta} \nabla \cdot\left(\tilde{p_{s}} V\right) d s\right) d \sigma d \zeta \\
& +\varepsilon\|\nabla V\|_{L^{2}(\Omega)}^{2}+\varepsilon\left\|V_{\zeta}\right\|_{L^{2}(\Omega)}^{2},
\end{aligned}
$$


and

$$
\begin{aligned}
& \frac{R}{c_{0}^{2}} \frac{d}{d t} \int_{\Omega}{\widetilde{p_{s}}}^{\prime 2} d \sigma d \zeta+\frac{R \mu_{2}}{c_{p} c_{0}^{2}} \int_{\Omega}\left|\nabla T^{\prime}\right|^{2} d \sigma d \zeta+\frac{R v_{2}}{c_{p} c_{0}^{2}} \int_{\Omega} \widetilde{p}_{s}\left(\frac{g \zeta}{R \widetilde{T}}\right)^{2}\left(\frac{\partial T^{\prime}}{\partial \zeta}\right)^{2} d \sigma d \zeta \\
& \quad+\left.\frac{k_{s 2} R}{c_{p} c_{0}^{2}} \int_{S^{2}} \widetilde{p_{s}}\left(\left(\frac{g \zeta}{R \widetilde{T}}\right)^{2} T^{\prime 2}\right)\right|_{\zeta=1} d \sigma \\
& \leq C\|V\|_{L^{2}(\Omega)}^{2}+C(M)\left(1+\left\|\nabla T_{2 \zeta}^{\prime}\right\|_{L^{2}(\Omega)}^{2}\right)\left\|T^{\prime}\right\|_{L^{2}(\Omega)}^{2} \\
& \quad-R \int_{\Omega} T^{\prime}\left(\frac{1}{\zeta} \int_{0}^{\zeta} \nabla \cdot\left(\widetilde{p_{s}} V\right) d s\right) d \sigma d \zeta \\
& \quad+\varepsilon\|\nabla V\|_{L^{2}(\Omega)}^{2}+\varepsilon\left\|V_{\zeta}\right\|_{L^{2}(\Omega)}^{2}+\varepsilon\left\|\nabla T^{\prime}\right\|_{L^{2}(\Omega)}^{2}+\varepsilon\left\|T_{\zeta}^{\prime}\right\|_{L^{2}(\Omega)}^{2} .
\end{aligned}
$$

Next, multiplying $(5.1)_{3}$ by $\widetilde{p}_{s} q$, we know

$$
\begin{aligned}
\frac{1}{2} \frac{d}{d t} & \int_{\Omega} \widetilde{p}_{s} q^{2} d \sigma d \zeta+\mu_{3} \int_{\Omega}|\nabla q|^{2} d \sigma d \zeta+v_{3} \int_{\Omega} \tilde{p}_{s}\left(\frac{g \zeta}{R \widetilde{T}}\right)^{2}\left|\frac{\partial q}{\partial \zeta}\right|^{2} d \sigma d \zeta \\
& +\left.k_{s 3} \int_{S^{2}} \widetilde{p_{s}}\left(\left(\frac{g \zeta}{R \widetilde{T}}\right)^{2} f\left(\left|V_{10}\right|\right) q^{2}\right)\right|_{\zeta=1} d \sigma \\
= & \frac{1}{2} \int_{\Omega} \frac{d \widetilde{p_{s}}}{d t} q^{2} d \sigma d \zeta-\int_{\Omega}\left(\widetilde{p}_{s} V_{1}^{*} \cdot \nabla q-\left(\int_{0}^{\zeta} \nabla \cdot\left(\widetilde{p}_{s} V_{1}^{*}\right)-\frac{\partial \widetilde{p_{s}}}{\partial t} \zeta\right) \frac{\partial q}{\partial \zeta}\right) q d \sigma d \zeta \\
& -\int_{\Omega}\left(V^{*} \cdot \nabla\right) q_{2} \widetilde{p}_{s} q d \sigma d \zeta+\int_{\Omega}\left(\int_{0}^{\zeta} \nabla \cdot\left(\widetilde{p}_{s} V^{*}\right) d s\right) \frac{\partial q_{2}}{\partial \zeta} q d \sigma d \zeta \\
& -\int_{\Omega} \delta_{21} \delta_{22}\left(\frac{1}{\zeta} \int_{0}^{\zeta} \nabla \cdot\left(\widetilde{p_{s}} V\right) d s\right) W(T) q d \sigma d \zeta \\
& +\int_{\Omega} \delta_{21} \delta_{22} \nabla \cdot\left(\widetilde{p}_{s} \bar{V}\right) W(T) q d \sigma d \zeta
\end{aligned}
$$

by applying (4.16), (4.24)-(4.26) and (4.45), we get

$$
\begin{aligned}
& -\int_{\Omega}\left(V_{1}^{*} \cdot \nabla q-\left(\frac{1}{\widetilde{p}_{s}} \int_{0}^{\zeta} \nabla \cdot\left(\widetilde{p}_{s} V_{1}^{*}\right)-\frac{\partial \widetilde{p}_{s}}{\partial t} \zeta\right) \frac{\partial q}{\partial \zeta}\right) \tilde{p}_{s} q d \sigma d \zeta=0 \\
& \left|\int_{\Omega}\left(V^{*} \cdot \nabla\right) q_{2} \widetilde{p}_{s} q d \sigma d \zeta\right| \\
& \quad \leq C \int_{\Omega}\left|V\|q\| \nabla q_{2}\right| d \sigma d \zeta \\
& \leq C\|V\|_{L^{4}(\Omega)}\|q\|_{L^{4}(\Omega)}\left\|\nabla q_{2}\right\|_{L^{2}(\Omega)} \\
& \leq C\|V\|_{L^{2}(\Omega)}^{\frac{1}{4}}\left(\|V\|_{L^{2}(\Omega)}+\|\nabla V\|_{L^{2}(\Omega)}+\left\|V_{\zeta}\right\|_{L^{2}(\Omega)}\right)^{\frac{3}{4}} \\
& \quad \times\|q\|_{L^{2}(\Omega)}^{\frac{1}{4}}\left(\|q\|_{L^{2}(\Omega)}+\|\nabla q\|_{L^{2}(\Omega)}+\left\|q_{\zeta}\right\|_{L^{2}(\Omega)}\right)^{\frac{3}{4}}\left\|\nabla q_{2}\right\|_{L^{2}(\Omega)} \\
& \leq C\|V\|_{L^{2}(\Omega)}^{2}+C\left(1+\left\|\nabla q_{2}\right\|_{L^{2}(\Omega)}^{8}\right)\|q\|_{L^{2}(\Omega)}^{2}+\varepsilon\|\nabla V\|_{L^{2}(\Omega)}^{2} \\
& \quad+\varepsilon\left\|V_{\zeta}\right\|_{L^{2}(\Omega)}^{2}+\varepsilon\|\nabla q\|_{L^{2}(\Omega)}^{2}+\varepsilon\left\|q_{\zeta}\right\|_{L^{2}(\Omega)}^{2} \\
& \leq C\|V\|_{L^{2}(\Omega)}^{2}+C(M)\|q\|_{L^{2}(\Omega)}^{2}+\varepsilon\|\nabla V\|_{L^{2}(\Omega)}^{2}+\varepsilon\left\|V_{\zeta}\right\|_{L^{2}(\Omega)}^{2}
\end{aligned}
$$


Lian and Ma Boundary Value Problems

(2020) 2020:103

Page 30 of 34

$$
\begin{aligned}
& +\varepsilon\|\nabla q\|_{L^{2}(\Omega)}^{2}+\varepsilon\left\|q_{\zeta}\right\|_{L^{2}(\Omega)}^{2}, \\
& \left|\int_{\Omega}\left(\int_{0}^{\zeta} \nabla \cdot\left(\widetilde{p}_{s} V^{*}\right) d s\right) \frac{\partial q_{2}}{\partial \zeta} d \sigma d \zeta\right| \\
& \leq C \int_{S^{2}}\left(\int_{0}^{1}\left(|V|^{2}+|\nabla V|^{2}\right) d \zeta\right)^{\frac{1}{2}}\left(\int_{0}^{1}\left|q_{2 \zeta}\right|^{2} d \zeta\right)^{\frac{1}{2}}\left(\int_{0}^{1}|q|^{2} d \zeta\right)^{\frac{1}{2}} d \sigma \\
& \leq C\left(\|V\|_{L^{2}(\Omega)}+\|\nabla V\|_{L^{2}(\Omega)}\right)\left(\int_{S^{2}}\left(\int_{0}^{1}\left|q_{2 \zeta}\right|^{2} d \zeta\right)^{2} d \sigma\right)^{\frac{1}{4}} \\
& \times\left(\int_{S^{2}}\left(\int_{0}^{1}|q|^{2} d \zeta\right)^{2} d \sigma\right)^{\frac{1}{4}} \\
& \leq C\left(\|V\|_{L^{2}(\Omega)}+\|\nabla V\|_{L^{2}(\Omega)}\right)\left(\int_{0}^{1}\left(\int_{S^{2}}\left|q_{2 \zeta}\right|^{4} d \sigma\right)^{\frac{1}{2}} d \zeta\right)^{\frac{1}{2}}\left(\int_{0}^{1}\right. \\
& \left.\times\left(\int_{S^{2}}|q|^{4} d \sigma\right)^{\frac{1}{2}} d \zeta\right)^{\frac{1}{2}} \\
& \leq C\left(\|V\|_{L^{2}(\Omega)}+\|\nabla V\|_{L^{2}(\Omega)}\right)\left(\int_{0}^{1}\left\|q_{2 \zeta}\right\|_{L^{2}\left(S^{2}\right)}\left\|q_{2 \zeta}\right\|_{H^{1}\left(S^{2}\right)} d \zeta\right)^{\frac{1}{2}} \\
& \times\left(\int_{0}^{1}\|q\|_{L^{2}\left(S^{2}\right)}\|q\|_{H^{1}\left(S^{2}\right)} d \zeta\right)^{\frac{1}{2}} \\
& \leq C\left(\|V\|_{L^{2}(\Omega)}+\|\nabla V\|_{L^{2}(\Omega)}\right)\left\|q_{2 \zeta}\right\|_{L^{2}(\Omega)}^{\frac{1}{2}}\left(\left\|q_{2 \zeta}\right\|_{L^{2}(\Omega)}^{\frac{1}{2}}+\left\|\nabla q_{2 \zeta}\right\|_{L^{2}(\Omega)}^{\frac{1}{2}}\right) \\
& \times\|q\|_{L^{2}(\Omega)}^{\frac{1}{2}}\left(\|q\|_{L^{2}(\Omega)}^{\frac{1}{2}}+\|\nabla q\|_{L^{2}(\Omega)}^{\frac{1}{2}}\right) \\
& \leq C(M)\left(1+\left\|\nabla q_{2 \zeta}\right\|_{L^{2}(\Omega)}^{2}\right)\|q\|_{L^{2}(\Omega)}^{2}+C\|V\|_{L^{2}(\Omega)}^{2} \\
& +\varepsilon\|\nabla V\|_{L^{2}(\Omega)}^{2}+\varepsilon\|\nabla q\|_{L^{2}(\Omega)}^{2}, \\
& \left|\int_{\Omega} \delta_{21} \delta_{22} \nabla \cdot\left(\tilde{p}_{s} \bar{V}\right) W(T) q d \sigma d \zeta\right| \\
& \leq C+C\|V\|_{L^{2}(\Omega)}^{2}+C\|\nabla V\|_{L^{2}(\Omega)}^{2}+C\|q\|_{L^{2}(\Omega)}^{2}, \\
& \left|\int_{\Omega} \delta_{21} \delta_{22}\left(\frac{1}{\zeta} \int_{0}^{\zeta} \nabla \cdot\left(\tilde{p}_{s} V\right) d s\right) W(T) q d \sigma d \zeta\right| \\
& \leq C+C\|V\|_{L^{2}(\Omega)}^{2}+C\|\nabla V\|_{L^{2}(\Omega)}^{2}+C\|q\|_{L^{2}(\Omega)}^{2} .
\end{aligned}
$$

By (5.6)-(5.10), we have

$$
\begin{aligned}
& \frac{d}{d t} \int_{\Omega} \tilde{p}_{s} q^{2} d \sigma d \zeta+\mu_{3} \int_{\Omega}|\nabla q|^{2} d \sigma d \zeta+\nu_{3} \int_{\Omega} \widetilde{p}_{s}\left(\frac{g \zeta}{R \widetilde{T}}\right)^{2}\left(\frac{\partial q}{\partial \zeta}\right)^{2} d \sigma d \zeta \\
& \quad+\left.k_{s 3} \int_{S^{2}} \widetilde{p}_{s}\left(f\left(\left|V_{10}\right|\right)\left(\frac{g \zeta}{R \widetilde{T}}\right)^{2} q^{2}\right)\right|_{\zeta=1} d \sigma \\
& \quad \leq C\|V\|_{L^{2}(\Omega)}^{2}+C(M)\left(1+\left\|\nabla q_{2 \zeta}\right\|_{L^{2}(\Omega)}^{2}\right)\|q\|_{L^{2}(\Omega)}^{2}+\varepsilon\|\nabla V\|_{L^{2}(\Omega)}^{2}+\varepsilon\left\|V_{\zeta}\right\|_{L^{2}(\Omega)}^{2} \\
& \quad+\varepsilon\|\nabla q\|_{L^{2}(\Omega)}^{2}+\varepsilon\left\|q_{\zeta}\right\|_{L^{2}(\Omega)}^{2} .
\end{aligned}
$$


Lian and Ma Boundary Value Problems

(2020) 2020:103

Page 31 of 34

Similarly, taking the inner product of $(5.1)_{4}$ with ${\tilde{p_{s}}}_{m}$, we get

$$
\begin{aligned}
\frac{1}{2} \frac{d}{d t} & \int_{\Omega}{\widetilde{p_{s}}}_{m_{w}}^{2} d \sigma d \zeta+\mu_{4} \int_{\Omega}\left|\nabla m_{w}\right|^{2} d \sigma d \zeta+v_{4} \int_{\Omega} \tilde{p_{s}}\left(\frac{g \zeta}{R \widetilde{T}}\right)^{2}\left|\frac{\partial m_{w}}{\partial \zeta}\right|^{2} d \sigma d \zeta \\
= & \frac{1}{2} \int_{\Omega} \frac{d \widetilde{p_{s}}}{d t} m_{w}^{2} d \sigma d \zeta \int_{\Omega}-\left(\widetilde{p_{s}}\left(V_{1}^{*} \cdot \nabla\right) m_{w}\right. \\
& \left.-\left(\int_{0}^{\zeta} \nabla \cdot\left(\widetilde{p_{s}} V_{1}^{*}\right)-\frac{\partial \widetilde{p_{s}}}{\partial t} \zeta\right) \frac{\partial m_{w}}{\partial \zeta}\right) m_{w} d \sigma d \zeta \\
& -\int_{\Omega}\left(V^{*} \cdot \nabla\right) m_{w 2} \tilde{p}_{s} m_{w} d \sigma d \zeta \\
& +\int_{\Omega} \frac{1}{\widetilde{p}_{s}}\left(\int_{0}^{\zeta} \nabla \cdot\left(\widetilde{p_{s}} V^{*}\right) d s\right) \frac{\partial m_{w 2}}{\partial \zeta} \tilde{p}_{s} m_{w} d \sigma d \zeta \\
& -\int_{\Omega} \delta_{21} \delta_{22}\left(\frac{1}{\zeta} \int_{0}^{\zeta} \nabla \cdot\left(\widetilde{p_{s}} V\right) d s\right) W(T) m_{w} d \sigma d \zeta \\
& +\int_{\Omega} \delta_{21} \delta_{22} \nabla\left(\widetilde{p_{s}} \cdot \bar{V}\right) W(T) m_{w} d \sigma d \zeta \\
& -\int_{\Omega} \delta_{21} \delta_{22} h_{1}\left(\left(\left(\frac{1}{\zeta} \int_{0}^{\zeta} \nabla \cdot\left(\widetilde{p_{s}} V\right) d s\right)+\nabla \cdot\left(\widetilde{p_{s}} \bar{V}\right)\right) W(T)\right) m_{w} d \sigma d \zeta,
\end{aligned}
$$

then using (4.16), (4.24)-(4.26) and (4.53), we obtain

$$
\begin{aligned}
& \int_{\Omega}-\left(\tilde{p}_{s}\left(V_{1}^{*} \cdot \nabla\right) m_{w}-\left(\int_{0}^{\zeta} \nabla \cdot\left(\tilde{p}_{s} V_{1}^{*}\right)-\frac{\partial \widetilde{p_{s}}}{\partial t} \zeta\right) \frac{\partial m_{w}}{\partial \zeta}\right) m_{w} d \sigma d \zeta=0, \\
& \left|\int_{\Omega}\left(V^{*} \cdot \nabla\right) m_{w 2} \widetilde{p}_{s} m_{w} d \sigma d \zeta\right| \\
& \leq C \int_{\Omega}|V \||\left|m_{w}\right| \nabla m_{w 2} \mid d \sigma d \zeta \\
& \leq C\|V\|_{L^{4}(\Omega)}\left\|m_{w}\right\|_{L^{4}(\Omega)}\left\|\nabla m_{w 2}\right\|_{L^{2}(\Omega)} \\
& \leq C\|V\|_{L^{2}(\Omega)}^{\frac{1}{4}}\left(\|V\|_{L^{2}(\Omega)}+\|\nabla V\|_{L^{2}(\Omega)}+\left\|V_{\zeta}\right\|_{L^{2}(\Omega)}\right)^{\frac{3}{4}} \\
& \times\left\|m_{w}\right\|_{L^{2}(\Omega)}^{\frac{1}{4}}\left(\left\|m_{w}\right\|_{L^{2}(\Omega)}+\left\|\nabla m_{w}\right\|_{L^{2}(\Omega)}+\left\|m_{w \zeta}\right\|_{L^{2}(\Omega)}\right)^{\frac{3}{4}}\left\|\nabla m_{w 2}\right\|_{L^{2}(\Omega)} \\
& \leq C\|V\|_{L^{2}(\Omega)}^{2}+C\left(1+\left\|\nabla m_{w 2}\right\|_{L^{2}(\Omega)}^{8}\right)\left\|m_{w}\right\|_{L^{2}(\Omega)}^{2}+\varepsilon\|\nabla V\|_{L^{2}(\Omega)}^{2} \\
& +\varepsilon\left\|V_{\zeta}\right\|_{L^{2}(\Omega)}^{2}+\varepsilon\left\|\nabla m_{w}\right\|_{L^{2}(\Omega)}^{2}+\varepsilon\left\|m_{w \zeta}\right\|_{L^{2}(\Omega)}^{2} \\
& \leq C\|V\|_{L^{2}(\Omega)}^{2}+C(M)\left\|m_{w}\right\|_{L^{2}(\Omega)}^{2}+\varepsilon\|\nabla V\|_{L^{2}(\Omega)}^{2} \\
& +\varepsilon\left\|V_{\zeta}\right\|_{L^{2}(\Omega)}^{2}+\varepsilon\left\|\nabla m_{w}\right\|_{L^{2}(\Omega)}^{2}+\varepsilon\left\|m_{w \zeta}\right\|_{L^{2}(\Omega)}^{2}, \\
& \left|\int_{\Omega}\left(\int_{0}^{\zeta} \nabla \cdot\left(\widetilde{p}_{s} V^{*}\right) d s\right) \frac{\partial m_{w 2}}{\partial \zeta} d \sigma d \zeta\right| \\
& \leq C \int_{S^{2}}\left(\int_{0}^{1}\left(|V|^{2}+|\nabla V|^{2}\right) d \zeta\right)^{\frac{1}{2}}\left(\int_{0}^{1}\left|m_{w 2 \zeta}\right|^{2} d \zeta\right)^{\frac{1}{2}}\left(\int_{0}^{1}\left|m_{w}\right|^{2} d \zeta\right)^{\frac{1}{2}} d \sigma \\
& \leq C\left(\|V\|_{L^{2}(\Omega)}+\|\nabla V\|_{L^{2}(\Omega)}\right)\left(\int_{S^{2}}\left(\int_{0}^{1}\left|m_{w 2 \zeta}\right|^{2} d \zeta\right)^{2} d \sigma\right)^{\frac{1}{4}}
\end{aligned}
$$


Lian and Ma Boundary Value Problems

(2020) 2020:103

Page 32 of 34

$$
\begin{aligned}
& \times\left(\int_{S^{2}}\left(\int_{0}^{1}\left|m_{w}\right|^{2} d \zeta\right)^{2} d \sigma\right)^{\frac{1}{4}} \\
& \leq C\left(\|V\|_{L^{2}(\Omega)}+\|\nabla V\|_{L^{2}(\Omega)}\right)\left(\int_{0}^{1}\left(\int_{S^{2}}\left|m_{w 2 \zeta}\right|^{4} d \sigma\right)^{\frac{1}{2}} d \zeta\right)^{\frac{1}{2}} \\
& \times\left(\int_{0}^{1}\left(\int_{S^{2}}\left|m_{w}\right|^{4} d \sigma\right)^{\frac{1}{2}} d \zeta\right)^{\frac{1}{2}} \\
& \leq C\left(\|V\|_{L^{2}(\Omega)}+\|\nabla V\|_{L^{2}(\Omega)}\right)\left(\int_{0}^{1}\left\|m_{w 2 \zeta}\right\|_{L^{2}\left(S^{2}\right)}\left\|m_{w 2 \zeta}\right\|_{H^{1}\left(S^{2}\right)} d \zeta\right)^{\frac{1}{2}} \\
& \times\left(\int_{0}^{1}\left\|m_{w}\right\|_{L^{2}\left(S^{2}\right)}\left\|m_{w}\right\|_{H^{1}\left(S^{2}\right)} d \zeta\right)^{\frac{1}{2}} \\
& \leq C\left(\|V\|_{L^{2}(\Omega)}+\|\nabla V\|_{L^{2}(\Omega)}\right)\left\|m_{w 2 \zeta}\right\|_{L^{2}(\Omega)}^{\frac{1}{2}}\left(\left\|m_{w 2 \zeta}\right\|_{L^{2}(\Omega)}^{\frac{1}{2}}+\left\|\nabla m_{w 2 \zeta}\right\|_{L^{2}(\Omega)}^{\frac{1}{2}}\right) \\
& \times\left\|m_{w}\right\|_{L^{2}(\Omega)}^{\frac{1}{2}}\left(\left\|m_{w}\right\|_{L^{2}(\Omega)}^{\frac{1}{2}}+\left\|\nabla m_{w}\right\|_{L^{2}(\Omega)}^{\frac{1}{2}}\right) \\
& \leq C(M)\left(1+\left\|\nabla m_{w 2 \zeta}\right\|_{L^{2}(\Omega)}^{2}\right)\left\|m_{w}\right\|_{L^{2}(\Omega)}^{2}+C\|V\|_{L^{2}(\Omega)}^{2} \\
& +\varepsilon\|\nabla V\|_{L^{2}(\Omega)}^{2}+\varepsilon\left\|\nabla m_{w}\right\|_{L^{2}(\Omega)}^{2}, \\
& \left|\int_{\Omega} \delta_{21} \delta_{22} \nabla \cdot\left(\widetilde{p_{s}} \bar{V}\right) W(T) m_{w} d \sigma d \zeta\right| \\
& \leq C+C\|V\|_{L^{2}(\Omega)}^{2}+C\|\nabla V\|_{L^{2}(\Omega)}^{2}+C\left\|m_{w}\right\|_{L^{2}(\Omega)}^{2}, \\
& \left|\int_{\Omega} \delta_{21} \delta_{22}\left(\frac{1}{\zeta} \int_{0}^{\zeta} \nabla \cdot\left(\widetilde{p_{s}} V\right) d s\right) W(T) m_{w} d \sigma d \zeta\right| \\
& \leq C+C\|V\|_{L^{2}(\Omega)}^{2}+C\|\nabla V\|_{L^{2}(\Omega)}^{2}+C\left\|m_{w}\right\|_{L^{2}(\Omega)}^{2} .
\end{aligned}
$$

Thanks to (5.13)-(5.17), we deduce

$$
\begin{aligned}
& \frac{d}{d t} \int_{\Omega} \widetilde{p}_{s} m_{w}^{2} d \sigma d \zeta+C \int_{\Omega}\left|\nabla m_{w}\right|^{2} d \sigma d \zeta+C \int_{\Omega}\left(\frac{\partial m_{w}}{\partial \zeta}\right)^{2} d \sigma d \zeta \\
& \quad \leq C(M)+C(M)\left(1+\left\|\nabla m_{w 2 \zeta}\right\|_{L^{2}(\Omega)}^{2}\right)\left\|m_{w}\right\|_{L^{2}(\Omega)}^{2}+\varepsilon\|\nabla V\|_{L^{2}(\Omega)}^{2}+\varepsilon\left\|V_{\zeta}\right\|_{L^{2}(\Omega)}^{2} \\
& \quad+\varepsilon\left\|\nabla m_{w}\right\|_{L^{2}(\Omega)}^{2}+\varepsilon\left\|m_{w \zeta}\right\|_{L^{2}(\Omega)}^{2} .
\end{aligned}
$$

Summing (5.3), (5.4), (5.11) and (5.18), we find that

$$
\begin{aligned}
& \frac{d}{d t} \int_{\Omega}{\widetilde{p_{s}}}_{s}\left(|V|^{2}+T^{\prime 2}+q^{2}+m_{w}^{2}\right)+R \frac{d}{d t} \int_{S^{2}} \frac{\widetilde{T}_{s}}{\widetilde{p}_{s}}\left(\int_{0}^{t} \nabla \cdot\left(\widetilde{p_{s}} \bar{V}\right) d \tau\right)^{2} d \sigma d \zeta \\
& \quad+C\|\nabla V\|_{L^{2}(\Omega)}^{2}+C\left\|\frac{\partial V}{\partial \zeta}\right\|_{L^{2}(\Omega)}^{2}+C\left\|\nabla T^{\prime}\right\|_{L^{2}(\Omega)}^{2}+C\left\|\frac{\partial T^{\prime}}{\partial \zeta}\right\|_{L^{2}(\Omega)}^{2} \\
& \quad+C\|\nabla q\|_{L^{2}(\Omega)}^{2}+C\left\|\frac{\partial q}{\partial \zeta}\right\|_{L^{2}(\Omega)}^{2}+C\left\|\nabla m_{w}\right\|_{L^{2}(\Omega)}^{2}+C\left\|\frac{\partial m_{w}}{\partial \zeta}\right\|_{L^{2}(\Omega)}^{2} \\
& \quad+\left.C \int_{S^{2}}\left(\left(f\left(\left|V_{1}\right|\right) V_{1}-f\left(\left|V_{2}\right|\right) V_{2}\right) \cdot\left(V_{1}-V_{2}\right)\right)\right|_{\zeta=1} d \sigma \\
& \quad+\left.C \int_{S^{2}} T^{\prime 2}\right|_{\zeta=1} d \sigma+\left.C \int_{S^{2}} q^{2}\right|_{\zeta=1} d \sigma
\end{aligned}
$$




$$
\begin{aligned}
\leq & C(M)\left(1+\left\|\nabla V_{2 \zeta}\right\|_{L^{2}(\Omega)}^{2}\right) \int_{\Omega} \tilde{p}_{s}|V|^{2} d \sigma d \zeta \\
& +C(M)\left(1+\left\|\nabla T_{2 \zeta}^{\prime}\right\|_{L^{2}(\Omega)}^{2}\right) \int_{\Omega} \widetilde{p}_{s} T^{2} d \sigma d \zeta \\
& +C(M)\left(1+\left\|\nabla q_{2 \zeta}\right\|_{L^{2}(\Omega)}^{2}\right) \int_{\Omega} \widetilde{p}_{s} q^{2} d \sigma d \zeta \\
& +C(M)\left(1+\left\|\nabla m_{w 2 \zeta}\right\|_{L^{2}(\Omega)}^{2}\right) \int_{\Omega} \widetilde{p}_{s} m_{w}^{2} d \sigma d \zeta,
\end{aligned}
$$

applying the Gronwall inequality, we can complete the proof.

\section{Acknowledgements}

The authors would like to express their heartful thanks to Prof. Hai-Liang Li for the useful comments and suggestions. The authors sincerely thank the reviewers for their valuable suggestions and useful comments that have led to the present improved version of the original manuscript.

\section{Funding}

The research is supported by the Key program of the National Natural Science Foundation of China No. 41630530 and the General Program of the National Natural Science Foundation of China No. 41975129.

\section{Availability of data and materials}

Data sharing not applicable to this article as no datasets were generated or analysed during the current study.

\section{Competing interests}

The authors declare that they have no competing interests.

\section{Authors' contributions}

All authors read and approved the final manuscript.

\section{Author details}

'School of Mathematics and Statistics, North China University of Water Resources and Electric Power, Zhengzhou, P.R. China. ${ }^{2}$ Institute of Atmospheric Physics, Chinese Academy of Sciences, Beijing, P.R. China. ${ }^{3}$ School of Mathematical Sciences, Capital Normal University, Beijing, P.R. China.

\section{Publisher's Note}

Springer Nature remains neutral with regard to jurisdictional claims in published maps and institutional affiliations.

Received: 21 October 2019 Accepted: 13 May 2020 Published online: 27 May 2020

\section{References}

1. Cao, C.S., Li, J.K., Titi, E.S.: Local and global well-posedness of strong solutions to the 3D primitive equations with vertical eddy diffusivity. Arch. Ration. Mech. Anal. 214(1), 35-76 (2014)

2. Cao, C.S., Li, J.K., Titi, E.S.: Global well-posedness of strong solutions to the 3D primitive equations with horizontal eddy diffusivity. J. Differ. Equ. 257(11), 4108-4132 (2014)

3. Cao, C.S., Li, J.K., Titi, E.S.: Global well-posedness of the three-dimensional primitive equations with only horizontal viscosity and diffusion. Commun. Pure Appl. Math. 69(8), 1492-1531 (2016)

4. Cao, C.S., Li, J.K., Titi, E.S.: Global well-posedness of the 3D primitive equations with horizontal viscosity and vertical diffusivity (2017) arXiv:1703.02512

5. Cao, C.S., Titi, E.S.: Global well-posedness and finite dimensional global attractor for a 3-D planetary geostrophic viscous model. Commun. Pure Appl. Math. 56(56), 198-233 (2003)

6. Cao, C.S., Titi, E.S.: Global well-posedness of the three-dimensional viscous primitive equations of large-scale ocean and atmosphere dynamics. Ann. Math. 166(1), 245-267 (2007)

7. Cao, C.S., Titi, E.S.: Global well-posedness of the 3D primitive equations with partial vertical turbulence mixing heat diffusion. Commun. Math. Phys. 310(2), 537-568 (2012)

8. Cao, C.S., Titi, E.S., Ziane, M.: A horizontal hyper-diffusion 3-D thermocline planetary geostrophic model: well-posedness and long-time behavior. Nonlinearity 17(5), 1749-1776 (2004)

9. Chepzhov, V.V., Vishik, M.l.: Attractors for Equations of Mathematical Physics. Am. Math. Soc., Providence (2002)

10. Guillén-González, F., Masmoudi, N., Rodríguez-Bellido, M.A.: Anisotropic estimates and strong solutions for the primitive equations. Differ. Integral Equ. 14(11), 1381-1408 (2001)

11. Guo, B.L., Huang, D.W.: Existence of weak solutions and the trajectory attractors for the moist atmospheric equations in geophysics. J. Math. Phys. 47, 083508 (2006)

12. Guo, B.L., Huang, D.W.: Existence of the universal attractor for the 3-D viscous primitive equations of large-scale moist atmosphere. J. Differ. Equ. 251(3), 457-491 (2011)

13. Hittmeir, S., Klein, R., Li, J.K., Titi, E.S.: Global well-posedness for the primitive equations coupled to nonlinear moisture dynamics with phase changes (2019) arXiv:1907.11199v1 
14. Huang, H.Y., Guo, B.L.: Existence of the solutions and the attractors for the large-scale atmospheric equations. Sci. China, Ser. D, Earth Sci. 49(6), 650-660 (2006)

15. Huang, H.Y., Guo, B.L.: The existence of weak solutions and the trajectory attractors to the model of climate dynamics. Acta Math. Sci. 27A(6), 1098-1110 (2007)

16. Lian, R.X., Zeng, Q.C.: Stability of weak solutions for the large-scale atmospheric equations. J. Inequal. Appl. 2014, 455 (2014)

17. Lian, R.X., Zeng, Q.C.: Existence of a strong solution and trajectory attractor for a climate dynamics model with topography effects. J. Math. Anal. Appl. 458(1), 628-675 (2018)

18. Lian, R.X., Zeng, Q.C., Jin, J.B.: Stability of weak solutions to climate dynamics model with effects of topography and non-constant external force. Sci. China Earth Sci. 61(1), 47-59 (2018)

19. Lions, J.L., Temam, R., Wang, S.H.: New formulations of the primitive equations of the atmosphere and applications. Nonlinearity 5(2), 237-288 (1992)

20. Lions, J.L., Temam, R., Wang, S.H.: Models Of The Coupled Atmosphere And Ocean (CAO I \& II). J. Odean Computational Mechanics Advances 1, Elsevier. 5-54, 55-1 19 (1993)

21. Lions, J.L., Temam, R., Wang, S.H.: Mathematical theory for the coupled atmosphere-ocean models (CAO III). J. Math. Pures Appl. 74(2), 105-163 (1995)

22. Pedlosky, J.: Geophysical Fluid Dynamics, 2nd edn. Springer, Berlin (1987)

23. Temam, R., Ziane, M.: Some mathematical problems in geophysical fluid dynamics. In: Handbook of Mathematical Fluid Dynamics, vol. III, pp. 535-657. North Holland, Amsterdam (2004)

24. Wu, Y.H., Mu, M., Zeng, Q.C., Li, Y.: Weak solutions to a model of climate dynamics. Nonlinear Anal., Real World Appl. 2(4), 507-521 (2001)

25. Zelati, M.C., Frémond, M., Temam, R., Tribbia, J.: The equation of the atmosphere with humidity and saturation: uniqueness and physical bounds. Physica D 264, 49-65 (2013)

26. Zelati, M.C., Huang, A.M., Kukavica, I., Temam, R., Ziane, M.: The primitive equations of the atmosphere in presence of vapor saturation. Nonlinearity 28(3), 625 (2014)

27. Zelati, M.C., Temam, R.: The atmospheric equation of water vapor with saturation. Boll. UMI 5, 309-336 (2012)

28. Zeng, Q.C.: Mathematical and Physical Foundations of Numerical Weather Prediction. Science Press, Beijing (1979) (in (hinese)

29. Zeng, Q.C.: A mathematic model of climate dynamics suitable for modern mathematical analysis. Sci. Atmos. Sin. 22(4), 408-417 (1998) (in Chinese)

\section{Submit your manuscript to a SpringerOpen ${ }^{\circ}$ journal and benefit from:}

- Convenient online submission

- Rigorous peer review

- Open access: articles freely available online

- High visibility within the field

- Retaining the copyright to your article

Submit your next manuscript at $\boldsymbol{~ s p r i n g e r o p e n . c o m ~}$ 